
internationales

vol. 22 - $n^{\circ} 3 \mid 2006$

Turquie 2006 : aux portes de l'Union européenne ?

\title{
Les voies internationales de la reproduction sociale
}

Trajectoires migratoires en grande bourgeoisie turque

\section{David Behar}

\section{(2) OpenEdition}

Journals

Édition électronique

URL : https://journals.openedition.org/remi/3261

DOI : 10.4000/remi.3261

ISSN : $1777-5418$

Éditeur

Université de Poitiers

Édition imprimée

Date de publication : 31 décembre 2006

Pagination : 39-78

ISBN : 978-2-911627-43-9

ISSN : 0765-0752

Référence électronique

David Behar, "Les voies internationales de la reproduction sociale ", Revue européenne des migrations internationales [En ligne], vol. 22 - $n^{\circ} 3$ | 2006, mis en ligne le 31 décembre 2009, consulté le 14 avril 2022. URL : http://journals.openedition.org/remi/3261 ; DOI : https://doi.org/10.4000/remi.3261

Ce document a été généré automatiquement le 14 avril 2022.

(c) Université de Poitiers 


\title{
Les voies internationales de la reproduction sociale
}

\author{
Trajectoires migratoires en grande bourgeoisie turque
}

David Behar

1 La situation étudiée traduit l'existence de mécanismes d'adaptation et de transmission spécifiques à la troisième génération de la grande bourgeoisie turque. L'histoire des familles qui composent la grande bourgeoisie d'aujourd'hui ne commence pas, dans bien des cas, avec la fondation de la République en $1923^{1}$, mais l'idée de troisième génération traduit bien les valeurs de ce groupe, qui a bâti sa légitimité dans ce nouveau contexte autour des principes kémalistes. L'appartenance à la grande bourgeoisie turque repose sur un passé collectif partagé par les familles qui ont fait partie de la première génération de la bourgeoisie républicaine, après la fondation de la République. Ces familles, dont la grande majorité sont installées à Istanbul, cumulent l'ensemble des capitaux permettant d'accéder au statut de dominant, à savoir le capital économique, social, culturel, scolaire et symbolique. Mais, plus que le montant de leur fortune, c'est la position qu'elles occupent à l'intérieur de ce milieu grand bourgeois qui importe réellement. Ainsi, l'ancienneté est référencée et contextualisée, une partie de l'histoire de la lignée étant délibérément extraite du discours de représentation des héritiers. Bien que les grands-pères des enquêtés soient eux-mêmes issus (directement ou par alliance) de lignées ottomanes, ils deviennent, dans la nouvelle République, les nouveaux patriarches. C'est à eux, en tant que doyens, que revient le droit de choisir le patronyme de la famille en 1934, tâche symbolique, il est vrai, mais dont on connaît l'importance dans la grande bourgeoisie et l'aristocratie (Pinçon et Pinçon-Charlot, 2000 ; Saint-Martin, 1993). Les familles de la grande bourgeoisie turque ayant réussi à maintenir leur position sont celles qui ont joué un rôle - au sein de l'État ou en tant qu'entrepreneur - dans les réformes républicaines, ou qui ont du moins accepté de renoncer à certains privilèges et de miser, en particulier, sur le capital scolaire.

2 La participation des héritiers aux mécanismes de la sélection scolaire, si elle permet une accumulation des capitaux, comporte aussi certains risques. Jean-Pierre Faguer explique ainsi que «la délégation à une institution scolaire d'une partie du métier de 
parent ne peut que produire des effets différents selon le pouvoir dont disposent les familles par rapport à l'institution scolaire » (Faguer, 1991). En choisissant de recourir à l'éducation internationale, les familles de la grande bourgeoisie turque ont donc sciemment accepté de réduire plus encore leur pouvoir de contrôle sur leurs héritiers, alors qu'ils disposaient localement - contrairement à d'autres pays de forte émigration étudiante - d'un système de formation performant des élites. Il existe plusieurs raisons à cela.

3 Cette décision est liée en premier lieu à l'ancienneté de l'entrée des héritiers turcs dans le champ de la compétition scolaire. Alors que, dans une frange importante des familles de la grande bourgeoisie française, la transmission du patrimoine n'impliquait pas, jusqu'à la seconde moitié du $\mathrm{xx}^{\mathrm{e}}$ siècle, la possession d'un diplôme (Bourdieu, Boltanski et Saint-Martin, 1973), les diplômes universitaires font partie intégrante des mécanismes de reproduction des élites turques depuis le $\mathrm{xIx}^{\mathrm{e}}$ siècle. Avant l'avènement de la République, les fonctions exclusivement administratives et militaires des élites ottomanes imposaient aux enfants de pachas de suivre une formation complète (Findley, 1989; Göçek, 1999). Puis, le développement d'une bourgeoisie d'affaires durant la période républicaine intervient dans le cadre des grands travaux commandés par l'État, nécessitant des hommes d'affaires qualifiés, en particulier des ingénieurs (Buğra, 1994 : 64 ; Göle, 1986) ${ }^{2}$.

4 Formés eux-mêmes pour une partie d'entre eux à l'étranger, ces chefs de famille accordaient donc une haute importance à la formation scolaire de leurs héritiers, quitte à renoncer à les avoir à leurs côtés durant plusieurs années. Néanmoins, la généralisation du recours à la voie internationale n'intervient qu'au début des années 1980. La Turquie sort alors de dix années de violence durant lesquelles le conflit entre la droite et la gauche avait atteint son paroxysme, dans les campagnes comme dans les villes, et en particulier dans les universités. Suite au Coup d'État militaire du 12 septembre 1980, le régime de "transition démocratique " (1980-1983) du Général Evren entreprend de réformer l'enseignement supérieur turc, tâche déléguée au nouveau Conseil de l'enseignement supérieur (YÖK). En 1983, la loi nº 1402 éloigne des universités un grand nombre de professeurs et de chercheurs soupçonnés d'entretenir des liens avec les mouvements politiques d'extrême gauche. Bien que favorables au retour de l'ordre, les familles de la grande bourgeoisie turque ne peuvent alors que constater une baisse de la qualité de l'enseignement, considérant de plus en plus l'éducation internationale comme la seule option d'excellence.

5 Enfin, l'arrivée au pouvoir du Partie de la Mère Patrie (ANAP) de Turgut Özal, qui restera au pouvoir durant dix ans (1983-1993), se traduit par l'institutionnalisation de la voie internationale. Bien que la création des premières universités privées au début des années 1990 offre désormais une option de rechange aux familles de la grande bourgeoisie, l'éducation internationale, encensée dans les médias, devient la voie reine pour la nouvelle génération d'héritiers. Face à la généralisation des migrations étudiantes au sein de l'élite scolaire, les héritiers de la grande bourgeoisie sont envoyés de plus en plus tôt et reviennent de plus en plus tard. À la compétition pour les formations internationales de complément se substitue la course aux formations internationales totales. En effet, les années 1980 sont aussi celles du développement d'un secteur privé indexé sur l'étranger. L'éducation internationale - et son ersatz, l'éducation en langue étrangère - devenant un mode de reproduction institutionnel, l'État turc possède désormais un contrôle limité sur la formation des élites. Dans ce contexte, 
la grande bourgeoisie turque possède la maîtrise de la reconversion des ressources de l'expérience internationale.

6 La généralisation du choix de l'éducation internationale dans les familles de la grande bourgeoisie turque à partir des années 1980 repose sur la confluence des stratégies familiales renvoyant à trois milieux bourgeois distincts. Dans le cas des enfants de diplomates et de hauts fonctionnaires internationaux, la socialisation internationale familiale constitue une forme spécifique d'apprentissage de la culture internationale, en particulier au travers des cycles migratoires quadriennaux imposés par la carrière des pères. Bien que ces familles entretiennent un réseau de sociabilité dense, illustré par la préférence pour le lycée français d'Ankara ou leur regroupement estival dans un même lieu de villégiature, les trajectoires des héritiers ne s'inscrivent pas dans un modèle de reproduction classique, le choix de la carrière diplomatique, en particulier, constituant davantage l'exception que la règle. Pour les héritiers des familles cosmopolites, c'est-à-dire dont les liens avec les élites d'autres pays ont été tissés au fil des générations, la socialisation internationale s'inscrit de longue date dans les stratégies familiales (Wagner, 2001). En revanche, le choix des destinations doit s'adapter aux exigences de la société du retour. Enfin, dans les familles dont les ancrages et les réseaux de sociabilité sont essentiellement nationaux, la préférence pour l'éducation internationale est liée davantage aux transformations du système éducatif turc et à l'évolution des critères du prestige relatif à l'expérience de l'étranger.

Les conclusions présentées reposent sur une enquête longitudinale d'une durée totale de trois ans. Une série d'entretiens semi-directifs et un travail sur leurs réseaux égocentrés ont été menés avec chacun des huit enquêtés de l'échantillon (cf. annexe pour les profils des enquêtés) durant les trois années suivant leur retour en Turquie. Il s'agit de 5 filles et 3 garçons, âgés de 25 à 30 ans, qui tous issus de familles appartenant à la grande bourgeoisie turque (le déséquilibre garçon/fille de l'échantillon est compensé au sein des réseaux, qui présentent une parité générale parfaite). Tous ont passé plus de 5 années à l'étranger (jusqu'à 15 ans pour certains), la majorité aux ÉtatsUnis. L'analyse de réseau a permis d'obtenir des données sur 1150 individus, les réseaux comprenant en moyenne 145 liens (l'échantillon a été constitué de manière à ce que très peu d'individus soient présents dans plus d'un réseau). Les listes de contacts ont été constituées essentiellement à partir de cahiers d'enregistrement des contacts quotidiens durant une semaine, du carnet d'adresses de téléphone portable et du carnet d'adresses e-mail. Les bases de données contiennent pour chaque contact les informations suivantes : données générales (sexe, âge, nationalité, profession, profession du père, état civil, niveau d'étude); données migratoires (nombre d'années passées à l'étranger, lieux de résidence successifs, date du retour); données relationnelles (ancienneté du contact, lieu de la rencontre, chaîne de contacts ayant permis la rencontre, fréquence des relations, nature du contact). $85 \%$ de l'ensemble des 1150 contacts sont de nationalité turque. Outre les proches parents, les réseaux sont constitués essentiellement d'amis, âgés de 23 à 33 ans ( $90 \%$ des contacts en moyenne). Ces réseaux d'amitié regroupent en moyenne $65 \%$ d'individus de nationalité turque ayant vécu plus d'un an à l'étranger, dont $83 \%$ plus de trois ans. On peut parler de réseaux d'héritiers, dans la mesure où, pour les contacts de nationalité turque de la même cohorte (âgés de 23 à 33 ans), l'homogénéité des origines sociales est très forte. Parmi les $80 \%$ de contacts dont la profession du père est connue par Ego, $57 \%$ sont des enfants de chefs d'entreprises, $20 \%$ de hauts fonctionnaires ou de diplomates, $10 \%$ de cadres dirigeants du secteur privé, $8 \%$ de professions libérales et $4 \%$ d'universitaires ${ }^{3}$. 
Des matrices de liaisons réciproques ont ensuite été constituées à partir de ces listes (Gribaudi, 1998). Cela a permis d'obtenir une représentation graphique des huit réseaux sur le logiciel PAJEK (De Nooy, Mrvar et Batagelj, 2005), donnant l'ensemble de la structure de ces réseaux, et sur laquelle les informations de la base de données peuvent être visualisées ${ }^{4}$. Les représentations graphiques ont ensuite été commentées par chaque enquêté au cours d'un long entretien. Cela a permis d'évoquer une par une les trajectoires des individus présents dans le réseau.

8 À partir des trajectoires migratoires des enquêtés et de celles des contacts de leurs réseaux de sociabilité respectifs, cet article propose une analyse d'un cycle migratoire, faisant l'objet d'une planification familiale, et dont l'enjeu est l'institution d'une cohorte d'héritiers dans la société d'origine. La concentration des destinations (1) et l'allongement de la durée migratoire (2) sont deux dynamiques spécifiques à cette cohorte. Elles participent de la nécessité de préparer au mieux le retour et de maîtriser l'évolution, en Turquie, des critères du prestige académique et professionnel internationaux. Néanmoins, la situation du retour (3), du fait de sa généralisation dans la cohorte étudiée, se traduit par une remise en question profonde de la relation entre projets individuels, cadres relationnels et cadres temporels. Elle vient contredire l'idée selon laquelle les jeunes héritiers ont achevé, une fois franchis les rites de passage, l'essentiel de leur processus de socialisation. Les difficultés de réadaptation ne sont compensées que par cette affinité particulière qu'est l'expérience de l'international en milieu bourgeois.

\section{Concentration des destinations}

Les migrations des héritiers s'inscrivent dans un mouvement plus général concernant l'ensemble des étudiants turcs, sachant que les héritiers sont en partie à l'origine de ce mouvement. Environ 50000 étudiants turcs suivent actuellement un cursus à l'étranger, soit $3,2 \%$ de l'ensemble des étudiants. Bien que ce pourcentage reste largement inférieur à ceux de plus petits pays voisins comme la Grèce $(13,3 \%)$ ou la Jordanie $(15,4 \%)$, les étudiants en provenance de Turquie constituent un flux majeur sur la carte des migrations étudiantes, du fait de son poids démographique. La Turquie occupe ainsi la $11^{\mathrm{e}}$ place du classement des pays pourvoyeurs d'étudiants migrants (UNESCO, 2005).

10 Les trajectoires migratoires des contacts des réseaux des enquêtés se distinguent du reste des migrations étudiantes. Très peu d'entre eux participent de la «fuite des cerveaux", dans la mesure où rares sont ceux qui projettent de s'installer définitivement à l'étranger. Ainsi, les succès académiques des héritiers sont salués dans les médias, tandis que le brain drain est montré comme un fléau national. Corrélativement, la voie internationale n'est pas une «fuite », parce qu'elle est héritée (éducation internationale des parents, voire des grands-parents; expérience familiale de l'international; double nationalité), ou du moins préparée (en famille; au travers d'intermédiaires privés; au travers des écoles d'été à l'étranger). Or, les réseaux migratoires se construisent précisément en fonction du degré de préparativité des futurs étudiants migrants - puis des futurs retournants. Plus le degré de préparativité est élevé, plus la superposition des trajectoires des individus issus d'un même milieu est importante. 


\section{Migrations étudiantes}

11 Depuis le XIX ${ }^{\mathrm{e}}$ siècle et les réformes de modernisation dites Tanzimat, nombreux futurs hauts fonctionnaires et académiciens ont bénéficié de bourses d'État destinées à financer une formation ou un complément de formation à l'étranger. Après l'avènement de la République, une Loi de 1929 relative aux demandes de départ à l'étranger («Ecnebi Memleketlere Gönderilecek Talebeler Hakkında Kanun») donne au Ministère de l'éducation la responsabilité du choix des candidats (Gençler et Çolak, 2003). Au total, 1472 étudiants ont ainsi été envoyés à l'étranger dans le cadre de cette loi. Certes, les taux de retour des boursiers d'État sont largement plus élevés que ceux des étudiants migrants ayant bénéficié d'une bourse de l'université étrangère où ils ont suivi leur cursus (Glaser et Habers, 1974). Néanmoins, il est intéressant de remarquer que la fuite des cerveaux concernait déjà les boursiers d'État, puisque près de $10 \%$ d'entre eux ne revinrent pas en Turquie. En 1987, une nouvelle loi donne également au nouveau Conseil de l'enseignement supérieur (YÖK) la possibilité d'envoyer des étudiants à l'étranger. Le YÖK prend cette fois des précautions contre le "non-retour ", puisque les boursiers sont contraints à travailler dans la fonction publique durant les 5 années suivant leur diplôme. Entre 1987 et 2002, 3600 bourses d'études à l'étranger seront délivrées, dont 1300 pour la seule année 1993, qui marqua la fin des " années Özal ». Rompant avec la tradition de coopération avec les universités d'Europe continentale (l'Allemagne et la France en particulier), le YÖK choisit d'envoyer la majeure partie des étudiants (91\%) dans des universités anglo-saxonnes : $49 \%$ aux États-Unis, $39 \%$ en Grande Bretagne et $2 \%$ au Canada. En même temps, cette politique est en accord avec celle menée au niveau national, visant à soutenir l'ouverture d'établissements privés anglophones.

En revanche, les flux généraux (bourses d'État + moyens privés) d'étudiants turcs à l'étranger sont plus équilibrés, comme le montre le tableau $n^{\circ} 1$. Bien que le nombre d'étudiants turcs (de nationalité turque) en Allemagne répertorié par l'UNESCO doive être revu à la baisse considérant qu'un nombre significatif d'entre eux y sont nés mais n'ont pas la nationalité allemande, la prééminence des universités anglo-saxonnes est moins évidente. Gençler et Çolak, en se basant sur les données de 2001 du Ministère de l'éducation $(\mathrm{MEB})^{5}$, relèvent ainsi que $26 \%$ des étudiants partant à l'étranger par leurs propres moyens ont choisi les États-Unis, mais que $21 \%$ d'entre eux ont opté pour l'Allemagne et $19,7 \%$ pour les universités des républiques turcophones d'Asie centrale. Ce premier constat révèle une première différence significative entre les trajectoires migratoires de l'ensemble des étudiants turcs suivant un cursus à l'étranger et celles des réseaux des huit enquêtés, qui sont, elles, concentrées sur les universités anglosaxonnes. Une raison importante à cela est le coût des études (coût de l'inscription et coût de la vie), bien plus élevé aux États-Unis ou en Angleterre que dans les Républiques turcophones ou encore en Allemagne, où les réseaux de parenté jouent un rôle important en termes de logement.

Tableau 1 : Principaux pays d'accueil des étudiants turcs

\begin{tabular}{|l|l|l|}
\hline Classement & Pays d'accueil & $\begin{array}{l}\text { Nombre d'étudiants } \\
\text { en 2002-2003 }\end{array}$ \\
\hline 1 & Allemagne & 27253 \\
\hline
\end{tabular}




\begin{tabular}{|l|l|l|}
\hline 2 & États-Unis & 11601 \\
\hline 3 & France & 2413 \\
\hline 4 & Autriche & 1820 \\
\hline 5 & Grande-Bretagne & 1411 \\
\hline 6 & Azerbaïdjan & 1376 \\
\hline 7 & Hollande & 780 \\
\hline 8 & Bulgarie & 738 \\
\hline 9 & Kirghizistan & 697 \\
\hline 10 & Suisse & 654 \\
\hline
\end{tabular}

Source : UNESCO, Statistiques éducation, 2005.

Toutefois, les États-Unis constituent un cas particulier parmi les destinations privilégiées par les étudiants turcs depuis les années 1980. Destination prisée aussi bien par les héritiers que par le reste des étudiants turcs, les flux d'étudiants turcs vers les États-Unis ont été multipliés par cinq entre 1985 et 2001 (cf. tableau $\mathrm{n}^{\circ}$ 2). Cela représente la plus importante augmentation mondiale sur cette période, même si elle s'inscrit dans un contexte général de croissance du nombre d'étudiants étrangers aux États-Unis, de 344000 en 1985 à 586000 en 2003. De 1985 à 2000, le nombre d'étudiants turcs aux États-Unis a été multiplié par quatre, passant de 2460 à 10100 étudiants. L'augmentation s'est poursuivie en 2001 et $2002(+8,7 \%$ en $2000 / 2001$ et $+10,1 \%$ en 2001/2002), s'arrêtant pour la première fois en 2003. Le nombre d'étudiants turcs a donc régressé cette année-là de $4 \%$, mais l'année universitaire 2004/2005 a montré les signes d'une reprise, le nombre d'étudiants turcs aux États-Unis passant à nouveau la barre des 12000 étudiants. La Turquie est la $8^{\mathrm{e}}$ nation d'origine représentée dans les universités américaines, derrière les 6 grands pays pourvoyeurs d'étudiants que sont l'Inde et la Chine (+ de 60000 étudiants), la Corée du Sud et le Japon (+ de 40000 étudiants), Taiwan et le Canada (+ de 20000 étudiants). La Turquie fait désormais partie du groupe des pays comptant plus de 10000 étudiants dans les universités américaines, au même titre que le Mexique, l'Indonésie et la Thaillande. Les étudiants turcs représentent $2 \%$ de l'ensemble des étudiants étrangers aux États-Unis, contre 0,7 \% en 1985 (cf. tableau n²).

\section{Réseaux d'héritiers migrants}

14 Le choix des destinations universitaires repose sur une combinaison de dynamiques individuelles, familiales et collectives, correspondant à une interprétation spécifique de la hiérarchie internationale du prestige académique et professionnel. La publication chaque année de classements internationaux répertoriant les universités les plus "performantes" sert davantage de caution aux choix des futurs étudiants que de mécanisme de sélection. Une fois franchi le cap des tests nationaux de sélection, chacun peut en effet faire ses propres choix et chaque université possède son propre système 
de sélection. Certes, la hiérarchie internationale du prestige académique et professionnel, qui se caractérise désormais par la prééminence du système américain, explique la "déviation » des flux internationaux d'étudiants de l'Europe vers les ÉtatsUnis. Néanmoins, une analyse plus détaillée des destinations (locations, universités, domaines d'études, etc.) révèle l'importance des dynamiques de réseau. L'évolution des trajectoires migratoires suit ainsi des jeux d'opportunités, des effets de mode ${ }^{6}$ et des effets de regroupement. Les jeux d'opportunités renvoient à la constitution à partir des années 1990 du marché des intermédiaires de l'éducation internationale, servant d'interface entre l'offre émanant des universités étrangères et la demande croissante des familles de la grande et de la petite bourgeoisie (les deux marchés étant distincts). Les effets de mode6 sont liés à l'idée que l'évolution des flux répond en partie aux principes de la diffusion verticale-descendante des goûts (trickle-down theory). Cette théorie, héritée des travaux de Veblen et Simmel sur la mode datant du début du siècle (Veblen, 1934 ; Simmel, 1957), fut reprise à partir des années 1950 par des sociologues parsoniens et par des économistes tels que Dwight Robinson (Robinson, 1961). Modélisation structuraliste de la diffusion des modes, la trickle-down theory considère qu'elles naissent au sommet de l'échelle sociale avant de descendre jusqu'aux classes moyennes, "les premières les abandonnant sitôt les secondes se préparent-elles à se les approprier " (Simmel, 1957). Bien que justement remise en cause par les sociologues de la mode contemporains, qui considèrent que certaines modes peuvent partir d'un groupe marginal (Erner, 2004), cette théorie s'applique bien au cas des destinations universitaires internationales. Les effets de regroupement, enfin, participent d'une multipolarisation des destinations étudiantes, en corrélation avec les dynamiques de réseau. La volonté d'une partie significative des jeunes héritiers migrants de retrouver une partie de leurs amis d'enfance également partis étudier à l'étranger entraîne la densification des flux migratoires en direction des universités de certaines zones (la côte Est américaine) et de certaines villes (Boston, New York, Londres, etc.). Les effets de regroupement sont corrélés aux deux premiers effets. Ils évoluent en effets de mode, et créent de nouvelles opportunités pour les promotions suivantes en attirant le regard de ces universités vers la Turquie.

Ainsi, dans le cas des étudiants, des effets de mode, dont l'analyse des réseaux égocentrés permet de retracer l'évolution, s'ajoutent au jeu des opportunités, qui concerne l'ensemble des migrations internationales. En effet, le jeu des opportunités a également aiguillé les flux des travailleurs migrants turcs en Allemagne, à partir de 1961, puis en France, au Benelux, en Autriche et en Suisse à partir de 1965. À propos de la diaspora turque en Europe, qui est estimée à près de 4 millions d'immigrés, de descendants d'immigrés, de naturalisés et de réfugiés politiques (Manço, 2004), Stéphane de Tapia parle ainsi d'une "multipolarisation de la migration turque ", les émigrés originaires d'une même région voire d'un même village se retrouvant dans les mêmes destinations (de Tapia, 1996). Bien évidemment, l'offre des universités étrangères n'est pas comparable avec, par exemple, l'appel de main-d'œuvre étrangère émis par l'Allemagne entre 1961 et 1973. Néanmoins, la multiplication à partir des années 1990 des intermédiaires privés intervenant sur les choix des étudiants a entraîné une densification des flux d'étudiants turcs sur certains pays, plus intéressés par le marché turc. 
Tableau 2 : Les étudiants turcs aux États-Unis depuis 1985

\begin{tabular}{|l|l|l|}
\hline Année & Nombre d'étudiants turcs & $\%$ du nombre total d'étudiants étrangers \\
\hline $2004 / 05$ & 12474 & 2,1 \\
\hline $2003 / 04$ & 11400 & 2,0 \\
\hline $2002 / 03$ & 11601 & 2,0 \\
\hline $2001 / 02$ & 12091 & 2,1 \\
\hline $2000 / 01$ & 10983 & 2,0 \\
\hline $1999 / 00$ & 10100 & 2,0 \\
\hline $1995 / 96$ & 7678 & 1,7 \\
\hline $1990 / 91$ & 4080 & 1,0 \\
\hline $1985 / 86$ & 2460 & 0,7 \\
\hline
\end{tabular}

Source : Open Doors, Institute of International Education, 2005

\section{Le choix de l'université}

Un grand nombre d'universités américaines, anglaises et canadiennes accueillant des étudiants turcs depuis plus de dix ans, disposent désormais d'inforimations précises sur le système scolaire turc. Tandis que les universités moins réputées se font connaître grâce aux bureaux de conseil ${ }^{7}$, les universités possédant une notoriété internationale sont en contact avec les conseillers d'orientation des meilleurs lycées privés de Turquie ${ }^{8}$. Le tableau $n^{\circ} 3$ reprend les dix universités ayant accepté le plus grand nombre d'anciens élèves du centre de préparation aux universités américaines TIPPS International, consacré à la préparation de l'examen de langue (Test of English as a Foreign Language, TOEFL), de l'examen d'entrée dans les universités américaines $(S A T)^{9}$ et des dossiers, spécifiques à chaque université, expliquant les motivations de l'élève. Ce centre, fondé en 1998, fut le premier du genre. Les prix pratiqués (plus de 100 dollars de l'heure pour des programmes de plus de 50 heures) ne sont envisageables que pour les familles les plus fortunées, mais c'est surtout le réseau de relations des fondateurs du centre qui leur a permis de maintenir une situation de quasi-monopole au sein de la grande bourgeoisie stambouliote ${ }^{10}$. Ces centres de préparation savent préparer à la fois les héritiers et, dans certains cas, leurs parents, à l'idée de partir étudier à l'étranger. Ils jouent également un rôle central dans la concentration des destinations.

Tableau 3 : Destinations des élèves de TIPPS International

\begin{tabular}{|l|l|l|}
\hline Université & Location & Nb d'élèves entrés \\
\hline Tufts & Boston (MA) & 32 \\
\hline
\end{tabular}




\begin{tabular}{|l|l|l|}
\hline Univ. of Virginia & Virginie & 31 \\
\hline Brown & Rhode Island & 26 \\
\hline Northwestern & Chicago (IL) & 26 \\
\hline Babson & Boston (MA) & 22 \\
\hline Parsons (art and design) & New York & 21 \\
\hline Franklin and Marshall & Pennsylvanie & 16 \\
\hline Univ. of Pennsylvania & Pennsylvanie & 16 \\
\hline Bentley & Boston (MA) & 15 \\
\hline Virginia Tech & Virginie & 14 \\
\hline Duke & Caroline du Nord & 13 \\
\hline Boston University & Boston (MA) & 13 \\
\hline Penn State & Pennsylvanie & 13 \\
\hline
\end{tabular}

Échantillon $=500$.

Source : www.tippsonline.com.

17 Sur le tableau $\mathrm{n}^{\circ} 3$, la concentration des choix des héritiers apparaît comme particulièrement forte. Bien que le centre ait des anciens élèves dans 120 universités américaines (sur plus de 4000 au total), 260 élèves, soit plus de la moitié des 500 anciens élèves de TIPPS (promotions 1998/1999 à 2004/2005), ont choisi les 13 mêmes universités. On peut ainsi avancer que la location de ces universités et les effets de mode liés à certaines d'entre elles ont au moins autant d'importance que leur classement dans les choix des élèves. En effet, si l'on se réfère au classement 2005 de l'US News and World Report, seules University of Pennsylvania et Duke figurent parmi les dix meilleures universités du pays (University of Virginia est 23e, mais elle est $n^{\circ} 1 \mathrm{du}$ classement des universités publiques). Si Northwestern ( $\left.n^{\circ} 12\right)$ et Brown ( $\left.n^{\circ} 15\right)$ sont classées parmi les 20 premières, Babson ou Bentley, par exemple, ne figurent pas parmi les 100 meilleures. Néanmoins, l'ampleur du nombre d'anciens élèves de TIPPS dans des universités qui restent tout de même très sélectives, telles que University of Pennsylvania, Brown, ou University of Virginia ${ }^{11}$, ne peut s'expliquer que par l'existence d'un intérêt réciproque entre ces universités et le centre. Pour donner un ordre de grandeur, les anciens élèves de TIPPS représentent à eux seuls près de $10 \%$ des 160 étudiants en premier et second cycles (undergraduate) originaires du Moyen-Orient et d'Afrique à l'Université de Pennsylvanie, et près de $5 \%$ de l'ensemble de la communauté internationale de Brown (500 étudiants en premier cycle) ${ }^{12}$. Et, outre Brown et Upenn, les autres universités de la prestigieuse Ivy League $e^{13}$, telles que Columbia (10 élèves), Yale (7), Harvard (5), et Cornell (5), ont accueilli au moins 5 anciens élèves de TIPPS. Alors qu'elles n'accueillaient qu'un ou deux élèves turcs par an dans les années 1990, ces universités, satisfaites des performances de ces étudiants, s'intéressent désormais de près au système éducatif turc. De leur côté, les professeurs 
de TIPPS savent désormais ce qui plaît au comité de sélection de ces universités, et incitent leurs élèves à préparer des dossiers de candidature pour ces universités. Les élèves, enfin, savent qu'ils retrouveront dans ces universités certains de leurs amis, et que la réputation en Turquie de ces établissements grandira à mesure que les diplômés rentreront en Turquie. La préparation à l'international des héritiers s'est donc progressivement organisée et institutionnalisée au cours des années 1990 grâce à l'émergence en Turquie d'un marché des études internationales. Les destinations des élèves de TIPPS révèlent une sédimentation de la multipolarisation des flux, accélérée par l'émergence d'un marché de la préparation à l'international qui joue sur les opportunités nées du regroupement des élèves dans certaines destinations, voire dans certaines universités. Mais, dans les années 1990 encore, c'est-à-dire la période durant laquelle les enquêtés ont fait leurs choix universitaires, la circulation de l'information se fait de manière informelle. Bien qu'au moins l'un des parents de 7 des 9 enquêtés ait suivi une partie de leur formation à l'étranger (cf. Tableau $\left.n^{\circ} 4\right)$, les choix universitaires des enfants exigent de se mettre à jour.

Tableau 4 : Éducation internationale des enquêtés et de leurs familles

\begin{tabular}{|c|c|c|c|c|}
\hline & Ego & Frère / Sœur & Mère & Père \\
\hline \multirow[t]{2}{*}{ Selin } & - BA* à Chicago & Sœur cadette : $\mathrm{X}$ & & \\
\hline & - MA* à Tel Aviv & & & \\
\hline \multirow[t]{4}{*}{ Eda } & - Collège à Birmingham & Frère cadet : & & \\
\hline & - BA à Londres & - BA/training & & \\
\hline & - MA à New York & à Washington & & \\
\hline & - Training* à Londres & & & \\
\hline \multirow[t]{3}{*}{ Gaye } & $\begin{array}{l}\text { - } 2 \text { ans à Hawaï entre } 2 \text { et } 4 \\
\text { ans }\end{array}$ & Sœur aînée : & - BA à New York & - AFS* à New York \\
\hline & - BA à Boston & - BA à Boston & & - PhD* à New York \\
\hline & & $\begin{array}{l}\text { - MBA*/training à } \\
\text { NY }\end{array}$ & & \\
\hline \multirow[t]{3}{*}{ Nesli } & - Primaire à Washington & & & - MA Tennessee \\
\hline & - BA en Pennsylvanie & Fille unique & & \\
\hline & - Training à New York & & & \\
\hline \multirow[t]{2}{*}{ Bilge } & - Primaire à Paris & & École de langues & \\
\hline & - Collège à Bruxelles & Fille unique & à Genève (Suisse) & \\
\hline
\end{tabular}




\begin{tabular}{|c|c|c|c|c|}
\hline & $\begin{array}{l}\text { - } \mathrm{BA} / \mathrm{MA} \text { et training à } \\
\text { Londres }\end{array}$ & & & \\
\hline \multirow[t]{2}{*}{ Kaan } & - BA à Washington & & - AFS à Baltimore & - AFS en Californie \\
\hline & - Training à New York & Fils unique & $\begin{array}{l}-\quad \text { MA à } \\
\text { Washington }\end{array}$ & $\begin{array}{l}-\quad \text { PhD à } \\
\text { Washington }\end{array}$ \\
\hline & - Training à San Francisco & & & \\
\hline \multirow[t]{3}{*}{ Murat } & - BA à Boston & Sœur aînée : & & \\
\hline & - Training à New York & - Langues à Florence & & \\
\hline & - Training à Londres & - Extension à Boston & & \\
\hline \multirow[t]{3}{*}{ Bulut } & - Primaire - Lycée à Paris & Sœur aînée : & & \\
\hline & - BA à Boston & - Collège à Paris & & - PhD à Paris \\
\hline & - Training à New York & - MA à Londres & & \\
\hline
\end{tabular}

* BA = Bachelor of Arts (appellation la plus fréquente pour le Bachelor's degree, équivalent à une maitrise, soit Bac +4$)$

MA = Master of Arts (appellation la plus fréquente pour le Master's degree, équivalent à un master, soit Bac + 6)

MBA = Master of Business Administration (Master tourné vers les professions du management) $\mathrm{PhD}=$ Doctor of Philosophy (équivalent à un Doctorat, soit Bac +9)

Training = Expérience professionnelle en entreprise à l'étranger

AFS = American Field Service (organisation non gouvernementale présente dans près de 60 pays, qui finance une année d'étude à l'étranger pour des lycéens en dernière année. La Turquie participe à ce programme depuis 1951).

\section{Dynamiques de réseaux}

La préférence de cette cohorte d'héritiers pour les universités anglo-saxonnes et, aux États-Unis, pour les universités de Boston et de New York, apparaît nettement sur le tableau $n^{\circ} 5$. Ce tableau présente, pour les huit réseaux, les trajectoires des contacts de nationalité turque ayant vécu au minimum un an hors de Turquie, soit un total de 597 liens. Les pourcentages ne sont pas cumulables, un individu pouvant avoir vécu dans plus d'un lieu, et chaque pourcentage traduit, pour l'ensemble des huit réseaux, le nombre de liens ayant vécu dans un lieu (ville, État, pays). Sachant que $87 \%$ des contacts de nationalité turque ayant vécu à l'étranger ont effectué leurs choix universitaires dans les années $1990^{14}$, c'est-à-dire avant l'explosion du marché des intermédiaires, l'analyse détaillée des trajectoires permet de mettre en évidence des dynamiques de réseau.

Tableau 5 : Destinations des contacts des huit réseaux

\begin{tabular}{|l|l|}
\hline États-Unis \\
\hline New York & $28 \%$ \\
\hline
\end{tabular}




\begin{tabular}{|l|l|}
\hline Boston & $18 \%$ \\
\hline Californie & $9 \%$ \\
\hline Washington & $7 \%$ \\
\hline Miami & $1 \%$ \\
\hline Canada & $1 \%$ \\
\hline Autres USA & $18 \%$ \\
\hline Europe - Asie - Moyen-Orient \\
\hline Grande-Bretagne & $25 \%$ \\
\hline France & $11 \%$ \\
\hline Allemagne & $5 \%$ \\
\hline Suisse & $4 \%$ \\
\hline Italie & $4 \%$ \\
\hline Bruxelles & $3 \%$ \\
\hline Autres Europe & $3 \%$ \\
\hline Israël & $2 \%$ \\
\hline Moyen-Orient & $3 \%$ \\
\hline Asie & $5 \%$ \\
\hline
\end{tabular}

19 Sur le graphe $n^{\circ} 1(1.1,1.2$ et 1.3$)$ sont représentées les trajectoires des liens de nationalité turque dans chacun des huit réseaux égocentrés. Le graphe $n^{\circ} 2$ opère le cumul des trajectoires des liens des huit réseaux. Pour faciliter la lecture, les trajectoires sont réparties sur deux graphes : " premiers départs et derniers retours » (2.1) et « pérégrinations » (2.2). 
Graphe $n^{\circ} 1.1$ : Trajectoires migratoires des contacts turcs de chaque réseau égocentré
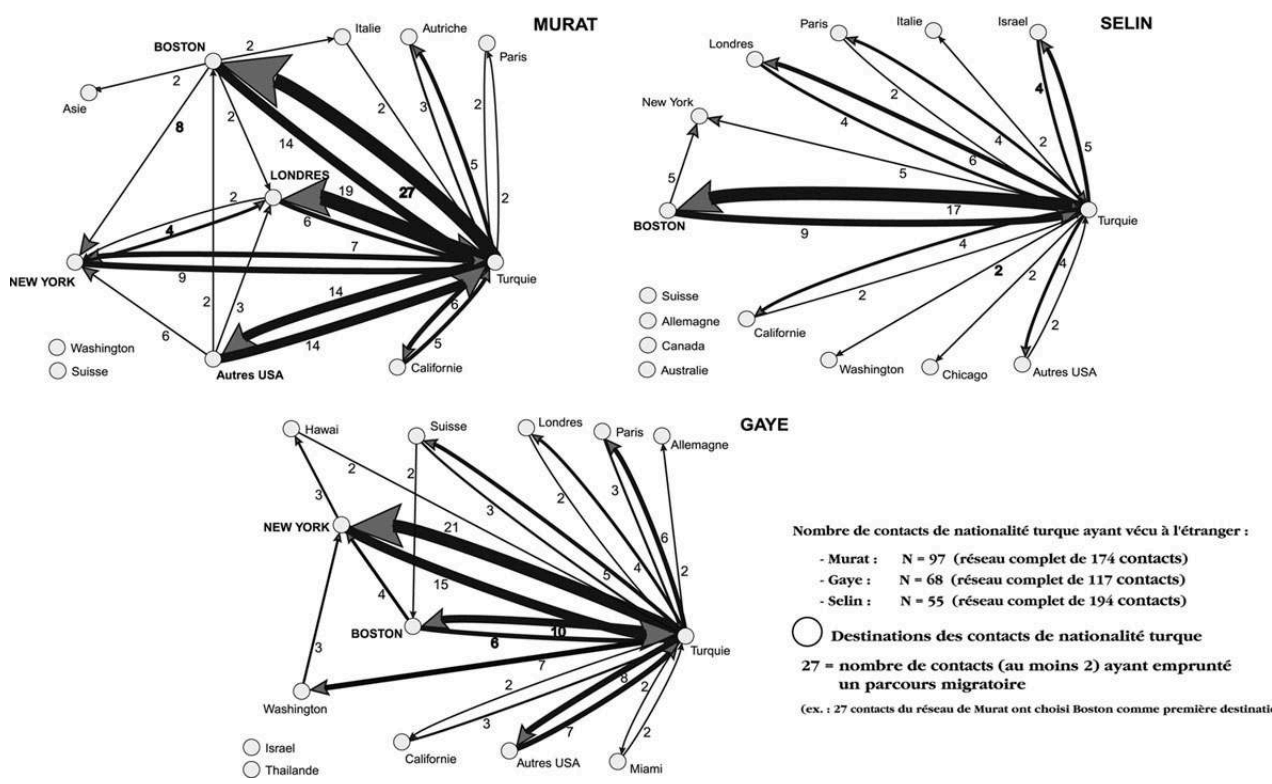

Graphe $n^{\circ} 1.2$ : Trajectoires migratoires des contacts turcs de chaque réseau égocentré
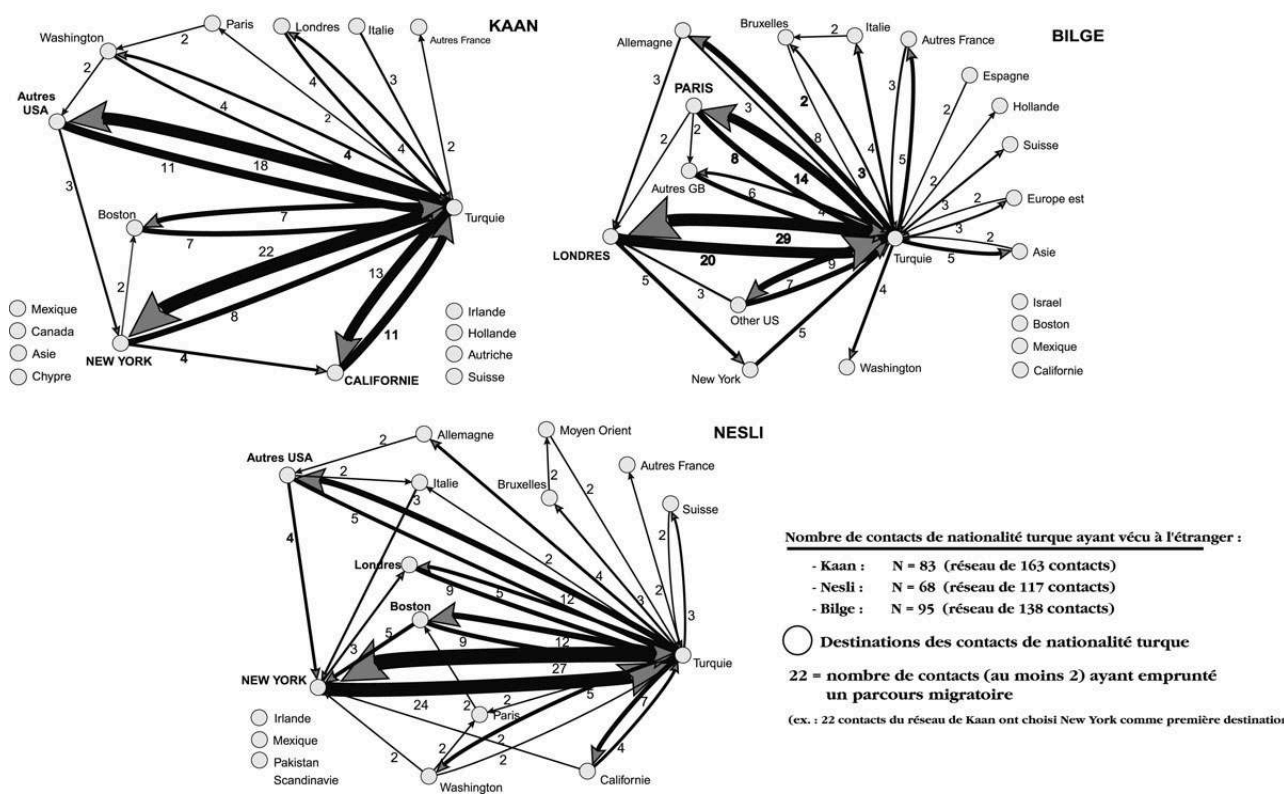
Graphe $\mathrm{n}^{\circ} 1.3$ : Trajectoires migratoires des contacts turcs de chaque réseau égocentré
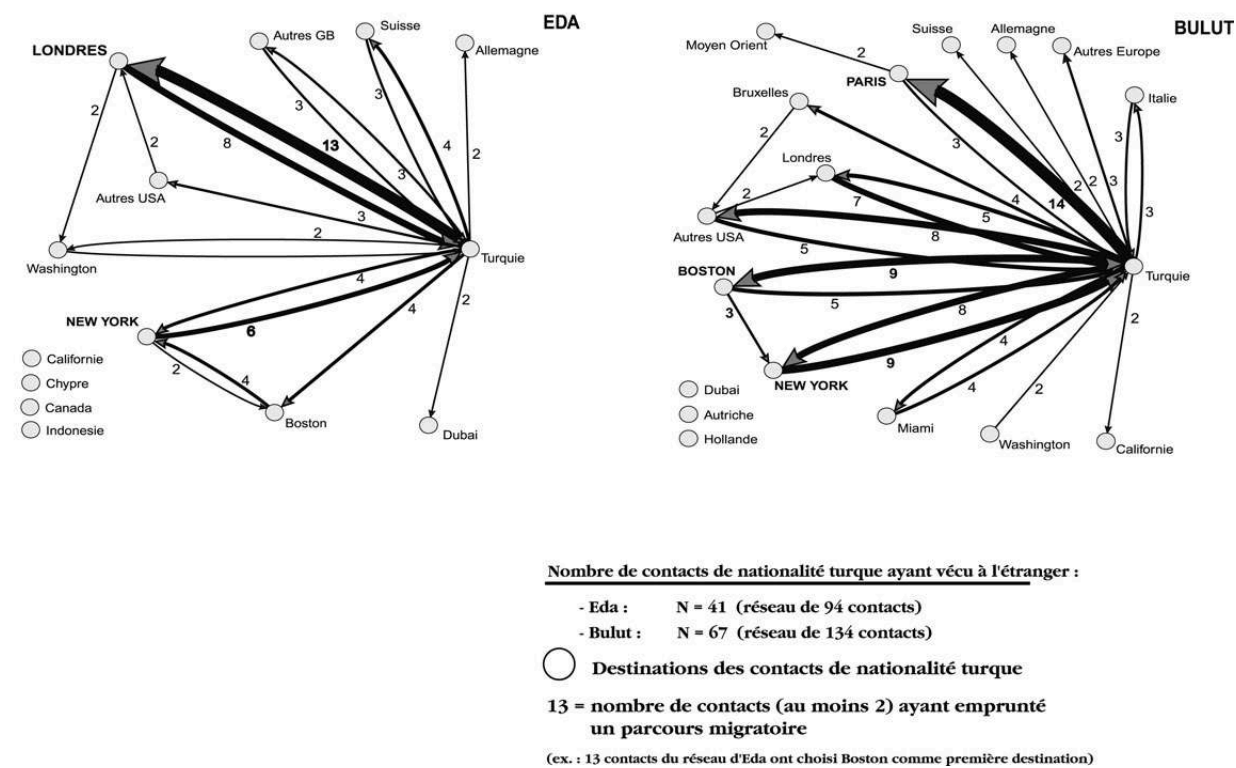

\section{Turquie $\rightarrow$ Boston}

Boston constitue l'une des destinations privilégiées dans la plupart des réseaux. Dans le cas des réseaux de Murat, Gaye et Bulut (cf. graphes 1.1 et 1.3), qui y ont tous les trois fait leurs études, l'ampleur des flux doit être rattachée à leur propre trajectoire. Néanmoins Boston figure également parmi les flux les plus importants dans les réseaux de Selin, Nesli et Kaan (graphes 1.1 et 1.2), bien qu'aucun d'entre eux n'y ait vécu. Dans le réseau de Selin, en particulier, un tiers (18/55) des contacts de nationalité turque ayant vécu à l'étranger ont passé au moins un an à Boston. Le graphe $\mathrm{n}^{\circ} 2$ permet d'identifier la place de Boston dans les trajectoires migratoires. Sur le graphe $\mathrm{n}^{\circ} 2.1$, Boston apparaît comme étant davantage une "première destination" (86/107 soit $80 \%$ ), principalement universitaire. Tandis qu'environ la moitié des contacts passés par Boston (51/107) ont choisi, comme Gaye, de rentrer en Turquie dès la fin de leurs études, l'autre moitié a migré vers une autre destination (sauf 8 contacts, qui se trouvent encore à Boston). Sur le graphe $n^{\circ} 2.2$, Boston est ainsi bien plus un lieu de partance qu'une seconde ou tierce destination. Outre vers Londres (4 contacs) et la Californie (4 contacts), c'est vers New York (30 contacts) que le flux de pérégrination est de loin le plus important. 
Graphe ${ }^{\circ} 2.1$ : "premiers départs et derniers retours"*

(ensemble des trajectoires migratoires des contacts turcs des huit réseaux égocentrés / $n=597$ )

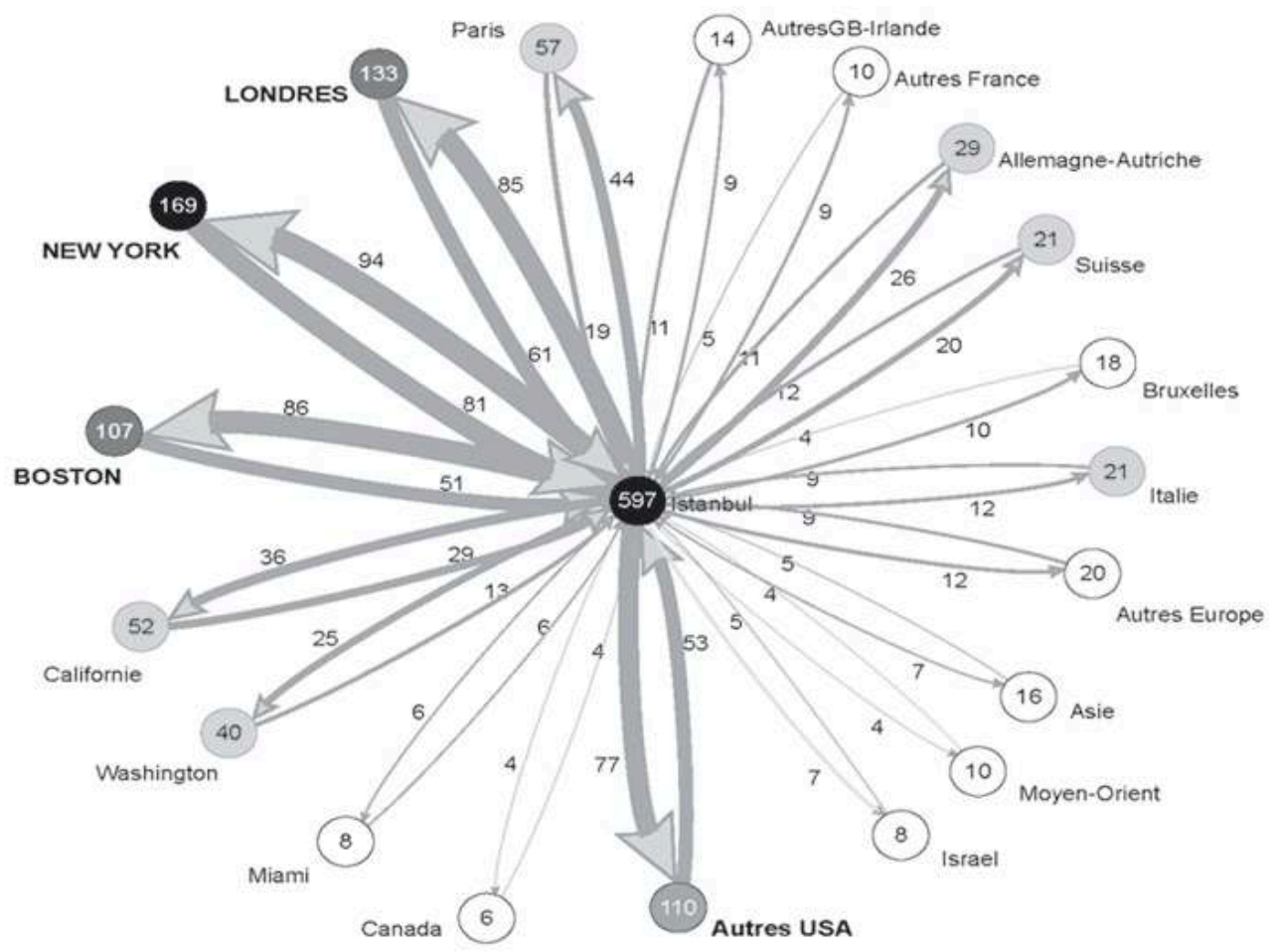

Graphe $\mathrm{n}^{\circ} 2.2$ : "pérégrinations"*

(ensemble des trajectoires migratoires des contacts turcs des huit réseaux égocentrés / $n=597$ )

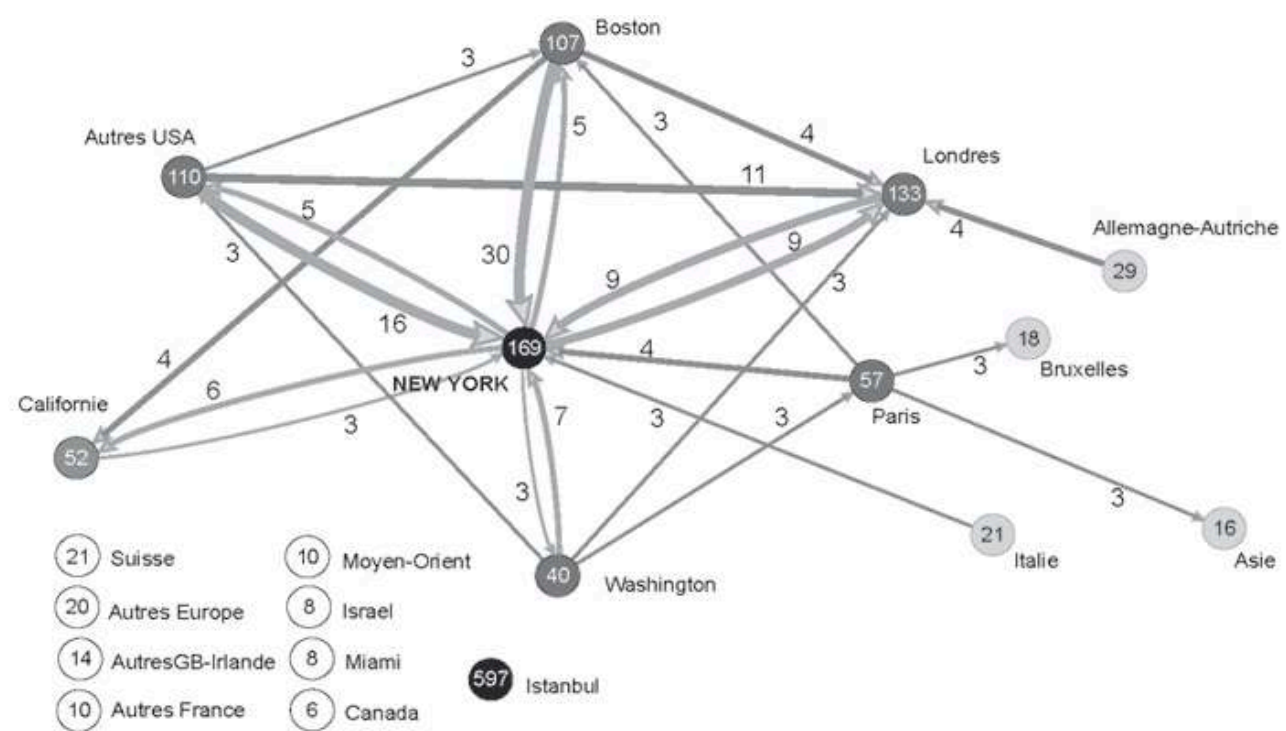

Pour plus de clarté, le graphe a été divisé en deux graphes. Sur le graphe $\mathrm{n}^{\circ} 2.2$ ne sont représentés que les trajets d'une destination étrangère à une autre (trajts empruntés par au minimum 3 contacts)

\section{Boston $\rightarrow$ New York}

6 des 8 réseaux comptent au moins 3 contacts ayant effectué ce parcours (Murat: 8 liens ; Selin : 5 ; Nesli : 5 ; Gaye : 4 ; Eda : 4 ; Bulut : 3). Murat, qui a lui-même suivi cette 
trajectoire, a été imité au total par 12 contacts de son réseau (4 sont étrangers), dont 9 sont des amis rencontrés à l'université. Comme lui, ces contacts ont prolongé leur séjour aux États-Unis dans le monde du travail - pour la plupart dans des domaines liés à la finance, New York constituant pour de jeunes diplômés une entrée prometteuse. Ces jeunes diplômés arrivent à New York avec derrière eux l'expérience de plusieurs années aux États-Unis. Ils ont été recrutés selon les mêmes critères scolaires que les étudiants américains, si bien que leur arrivée à New York participe d'avantage d'un mouvement de recentrage que d'une migration. Le recentrage postuniversitaire vers New York ne concerne d'ailleurs pas uniquement Boston. En tout 26 contacts ont ainsi vécu à New York après avoir étudié dans une autre ville américaine (16 'Autres USA' +7 'Washington' + 3 'Californie')

\section{Turquie $\leftrightarrow$ New York}

Cumulant destinations universitaires et professionnelles, New York a attiré près d'un tiers $(28 \%)$ de l'ensemble des contacts de nationalité turque ayant vécu à l'étranger (cf. tableau $n^{\circ} 5$ ). Sept des huit réseaux comptent au minimum $20 \%$ de contacts ayant vécu à New York (les destinations du réseau de Bilge sont essentiellement européennes). Au sein des 5 réseaux où New York figure dans la trajectoire d'Ego, les flux passant par New York sont supérieurs à la moyenne générale $(>28 \%)$, sauf dans les réseaux de Nesli et de Murat. Une explication à cela est la durée du séjour à New York. Tandis que Nesli y a travaillé 4 ans et Kaan 3 ans, Murat n'y a séjourné que 2 ans, et Bulut et Eda qu' 1 an. $50 \%(45 / 89)$ des contacts turcs ayant vécu à l'étranger du réseau de Nesli sont passés par New York, et $31 \%(26 / 83)$ de ceux du réseau de Kaan, contre $25 \%(24 / 97)$ pour le réseau de Murat, $22 \%$ (15/67) dans celui de Bulut et $21 \%$ (9/41) dans celui d'Eda. Dans les 3 réseaux où New York ne figure pas dans la trajectoire d'Ego, les pourcentages sont logiquement inférieurs à la moyenne générale (Selin : 11/55 soit $20 \%$; Bilge : 9/97 soit $10 \%)$, à l'exception des contacts du réseau de Gaye (30/68 soit $44 \%)$. Ce pourcentage très élevé s'explique en partie par le fait que la sœur aînée de Gaye y a vécu pendant plus de 5 ans. Gaye rendait ainsi fréquemment visite à sa sœur à New York durant ses études à Boston. Néanmoins, seuls 2 des 30 contacts de son réseau y ayant vécu ont été activés à New York, contre 3 à Boston et 25 à Istanbul. Parmi les 25 contacts activés à Istanbul, 5 ont été introduits par sa sœur après le retour de Gaye, 4 sont des amis de la famille connus depuis l'enfance, et 7 sont des amis d'enfance connus à l'école primaire ou au lycée. Cela montre bien l'effet des dynamiques de réseau sur le choix des destinations. Au sein du réseau familial de Gaye et de son réseau d'amis du lycée, New York constitue une destination privilégiée. De même, dans le réseau de Nesli, qui a pourtant vécu 4 ans à New York, seuls $35 \%$ (16/45) des contacts turcs y ayant séjourné ont été activés à New York. Les autres contacts ont été activés pour un tiers (10/29) par la famille durant l'enfance (à Washington, Ankara et Istanbul), pour un tiers (9/29) durant les années passées à l'étranger par l'intermédiaire d'amis d'enfance lors des retours estivaux, et pour un tiers (11/29) à Istanbul après son retour.

Enfin, il reste à évaluer New York en tant que première et dernière destination. Sur le graphe $\mathrm{n}^{\circ} 2.1$, New York apparaît comme la principale destination des «premiers départs » (94/597 contacts, soit ${ }^{15} \%$ de l'ensemble des premières destinations des liens turcs ayant vécu à l'étranger). Dans les réseaux de Nesli et de Gaye, en particulier, New York est la première destination de près d'un tiers des contacts de nationalité turque ayant vécu à l'étranger. New York est également la principale « dernière destination » 
( $81 / 400$ soit $20 \%$ des contacts rentrés en Turquie). Cette ville constitue dans de nombreux cas le terminus des pérégrinations. Dans les réseaux de Nesli et de Gaye, New York est ainsi la dernière destination de plus de la moitié des retournants de nationalité turque ayant vécu à New York (27/45 soit $60 \%$ dans le réseau de Nesli, et $15 / 30$ soit $50 \%$ dans le réseau de Gaye). En revanche, les pourcentages tombent autour de $30 \%$ dans les réseaux de Kaan (8/26) et de Murat (8/24), qui ont tous deux poursuivi leurs pérégrinations après avoir quitté New York, le premier à San Francisco et le second à Londres. La trajectoire d'Ego doit donc là aussi être prise en compte dans l'interprétation des flux des contacts composant les réseaux (4 contacts du réseau de Kaan ont également rejoint la Californie et 4 contacts du réseau de Murat sont partis à Londres après avoir vécu à New York).

\section{Turquie $\leftrightarrow$ Londres} contacts de nationalité turque des huit réseaux égocentrés. 133 des 597 contacts de l'échantillon considéré, soit $22 \%$ ( $25 \%$ si l'on ajoute les autres villes britanniques, cf. tableau n ${ }^{\circ} 5$ ), ont vécu au minimum une année à Londres. Mais, contrairement à New York, Londres n'est fortement représentée que dans les réseaux des 3 enquêtés qui y ont vécu. Ainsi, les contacts des réseaux d'Eda (24/41 soit 59\%), Bilge (45/97 soit $46 \%$ ) et Murat (28/97 soit $29 \%$ ) composent l'essentiel des trajectoires passant par Londres, les 6 autres réseaux ne comptant qu'entre $6 \%$ et $12 \%$ de contacts ayant vécu à Londres. Il est néanmoins possible d'identifier certaines dynamiques de réseau :

- $80 \%(36 / 45)$ des contacts du réseau de Bilge ayant vécu à Londres ont été activés en Angleterre, mais $20 \%$ d'entre eux sont issus de familles de diplomates. Outre les séjours à Londres liés à la carrière des pères, la préférence de ces familles pour les destinations européennes transparaît clairement. Sur les 26 contacts du réseau de Bilge dont les pères sont des diplomates, 7 ont choisi d'étudier à Londres et 4 en France, la seule destination importante outre atlantique étant New York (3 liens).

- $68 \%(19 / 28)$ des contacts du réseau de Murat ayant vécu à Londres ont été activés en Angleterre, mais c'est au travers de 2 amis d'enfance et de 7 amis rencontrés à Boston et à New York que Murat rencontre l'essentiel de ses fréquentations londoniennes.

- 33 \% seulement des contacts du réseau d'Eda ayant vécu à Londres ont été activés en Angleterre, l'essentiel étant des amis d'enfance (de quartier et d'école) et des parents (deux cousins et un oncle). Comme New York dans le réseau de Gaye, Londres apparaît être une destination privilégiée dans les familles du réseau d'Eda, qui fut d'ailleurs envoyé en Angleterre dès l'école primaire.

Enfin, Londres est, au même titre que New York, à la fois l'une des principales "première " et "dernière destination ». La moindre importance du flux Londres $\rightarrow$ Istanbul (cf. graphe $\mathrm{n}^{\circ}{ }^{2.1}$ ) est liée au nombre important de contacts n'étant pas encore rentrés en Turquie au moment de la constitution des listes de contacts (la majeure partie d'entre eux sont rentrés depuis). Comme New York encore, Londres est un lieu de recentrage, où viennent travailler de jeunes diplômés aux États-Unis (18 contacts en tout en cumulant " autres USA », Boston et Washington - cf. graphe $n^{\circ} 2.2$ ). Une fois recentrés, ces jeunes professionnels peuvent éventuellement compléter leur expérience professionnelle en quittant Londres pour New York ( 9 contacts donc 5 dans le réseau de Bilge) ou, dans l'autre sens, New York pour Londres ( 9 contacts dont 4 dans le réseau de Murat, qui a lui-même suivi cette trajectoire).

Revue européenne des migrations internationales, vol. 22 - n³| 2006 
L'analyse des trajectoires des contacts des huit réseaux égocentrés a révélé l'importance de la circulation informelle des informations relatives aux formations internationales avant la mise en place de ce marché. Certes, l'échantillon n'est pas représentatif de l'ensemble des héritiers formés à l'étranger, dès lors que la trajectoire d'Ego est logiquement corrélée à celles des contacts de son réseau (en particulier pour les contacts activés à l'étranger). Néanmoins, il est tout de même possible de distinguer des flux liés directement aux différents milieux bourgeois : la préférence pour New York spécifique aux réseaux familiaux de Nesli et de Gaye; la préférence pour Londres spécifique au réseau d'Eda ; la préférence pour Boston des anciens élèves du prestigieux lycée américain Robert College, dont Gaye et Selin sont diplômées ; la préférence enfin pour les destinations européennes spécifiques à la communauté des diplomates, Bulut constituant, comme il l'explique lui-même, une « exception ».

Par ailleurs, l'ordre des destinations dans les pérégrinations des héritiers apporte également des informations sur la fonction de ces destinations. Le regroupement des héritiers dans les universités de Boston a fait de cette ville une "première destination ", à partir de laquelle il est possible de se recentrer vers New York ou Londres, qui apparaissent comme les deux grands pôles d'attraction. Ces deux villes, qui incarnent l'excellence professionnelle aux yeux des familles de la grande bourgeoisie turque (compétitivité du marché, prestige et visibilité internationale des grandes firmes, etc.), sont le terrain d'entraînement des héritiers. Elles sont les principaux lieux de « l'allongement de la migration».

\section{Allongement de la durée migratoire}

La banalisation de l'expérience internationale incite à partir plus tôt et de revenir de plus en plus tard. Cette quête d'une formation internationale totale se traduit par des départs de plus en plus précoces et des retours de plus en plus tardifs.

\section{Durées migratoires}

L'échantillon procuré par les 8 réseaux égocentrés donne un aperçu de la nature et de la durée des formations universitaires internationales suivies par les héritiers. Sur le graphe $\mathrm{n}^{\circ} 3$ (3.1, 3.2 et 3.3) sont représentées les durées migratoires de l'ensemble des contacts de chaque réseau. En moyenne, $65 \%$ des contacts de nationalité turque ont vécu au moins un an à l'étranger. Mis à part le réseau de Selin où une minorité de ces contacts ont étudié ou travaillé à l'étranger ( $40 \%$ ) et le réseau de Bilge où, à l'inverse, la quasi-totalité des contacts ont vécu hors de Turquie au moins un an (90\%), les autres réseaux comptent entre 60 et $75 \%$ de liens possédant une expérience de l'étranger. 
Graphe $n^{\circ} 3.1$ : Nombre d'années passées à l'étranger par les contatcs de chaque réseau égocentré

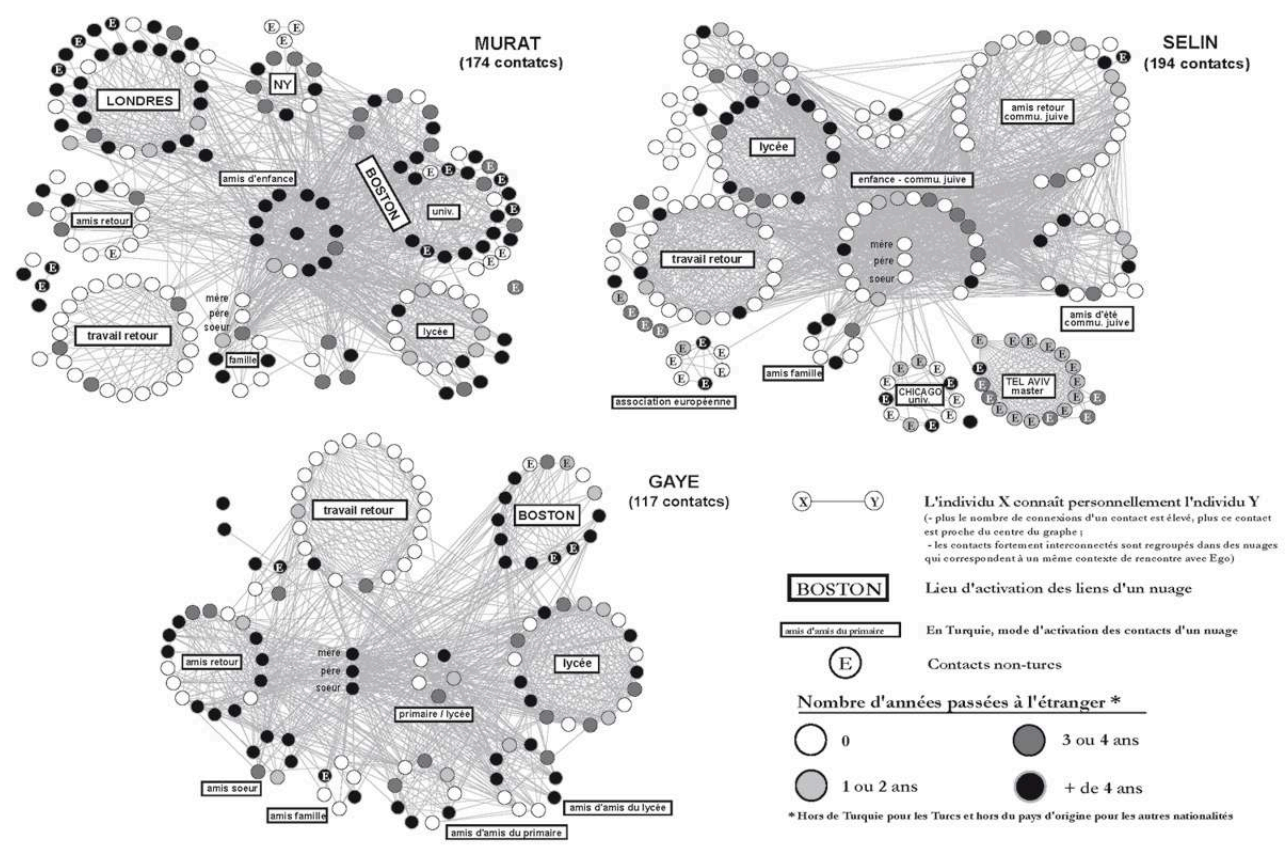

Graphe n`3.2: Nombre d'années passées à l'étranger par les liens de chaque réseau égocentré
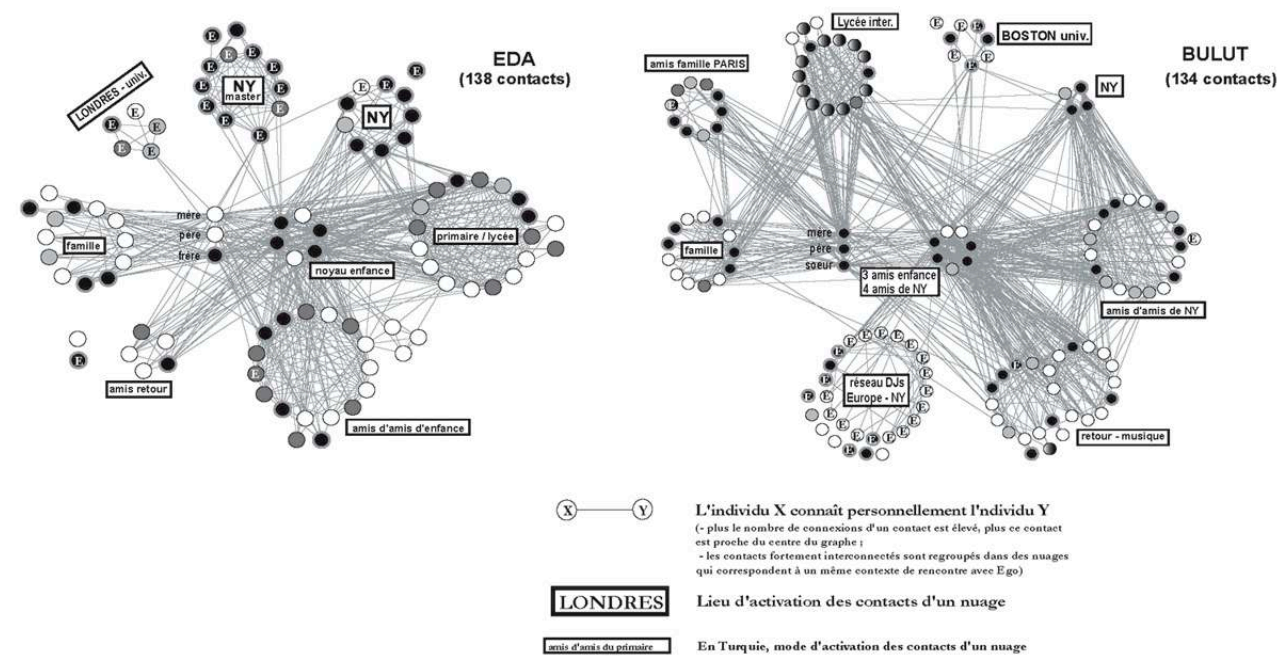

(E) Contacts non-tures

Nombre d'années passées a l l'étranger *
1 ou 2 ans


Graphe $n^{\circ} 3.3$ : Nombre d'années passées à l'étranger par les contatcs de chaque réseau égocentré
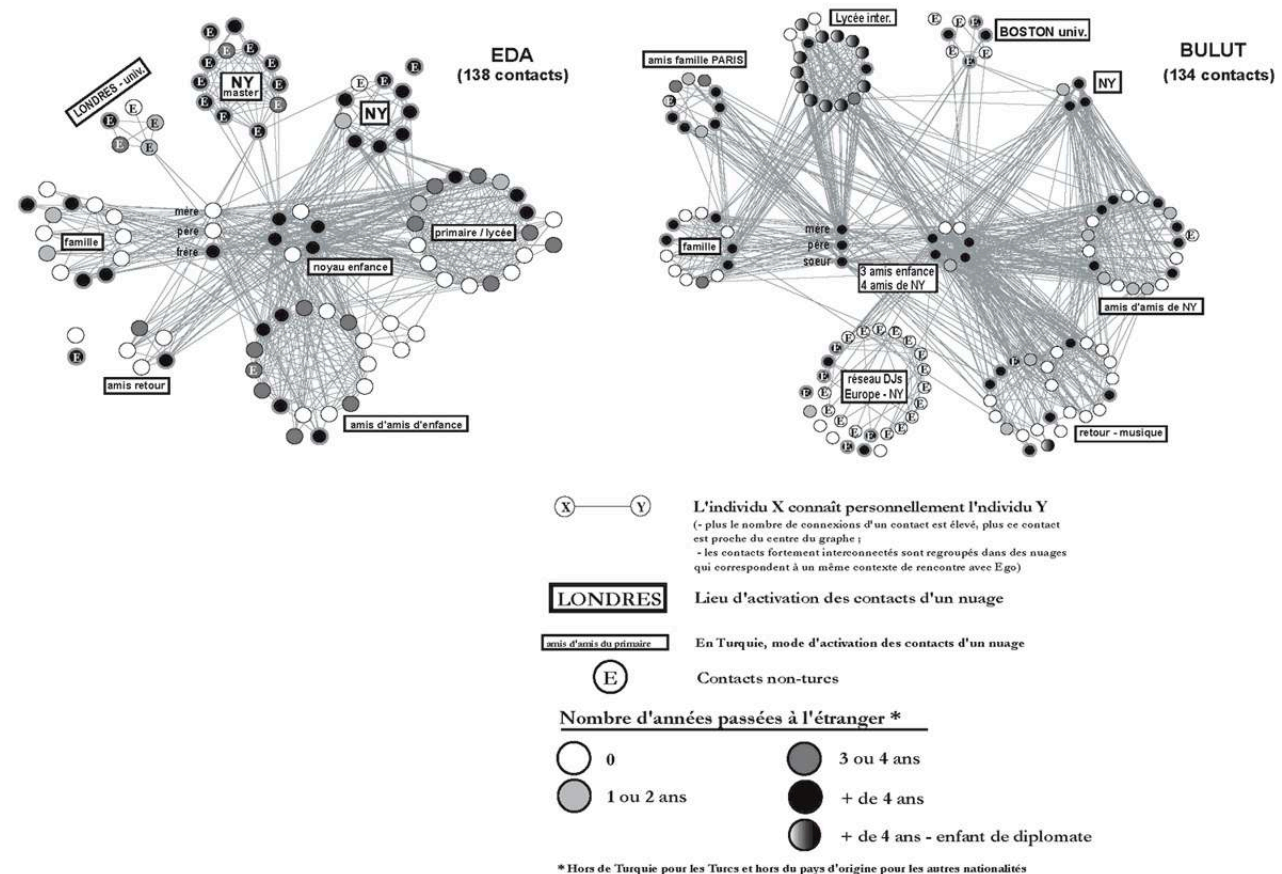

Ces représentations graphiques permettent de mettre en évidence les contacts existant entre la structure et l'évolution des réseaux de sociabilité d'une part, et les rapports à l'international spécifiques à chaque cercle de fréquentations d'autre part. Le positionnement des points correspond au départ à un algorithme appelé Fruchterman Reingold, qui calcule la position de chaque point de manière à ce que la distance entre un point et l'ensemble de ses connexions soit la plus courte possible. Les contacts les plus interconnectés sont attirés vers le centre du graphe, dans le noyau du réseau, et les contacts possédant très peu de connections sont rejetés à la périphérie du réseau. Puis, pour plus de lisibilité, les contacts fortement interconnectés ont été regroupés à l'intérieur de nuages, correspondant à un même contexte de rencontre avec Ego. Enfin, à l'aide des données migratoires de la base de données, une partition «durée migratoire " a été introduite. Elle distingue les contacts n'ayant jamais vécu hors de leur pays de naissance, les contacts qui ont vécu 1 ou 2 ans à l'étranger (durée correspondant à une formation linguistique ou à un mastère), ceux qui ont vécu 3 ou 4 ans à l'étranger (durée correspondant à l'obtention d'un diplôme universitaire de second cycle) ${ }^{16}$, ceux qui ont vécu (seuls) plus de quatre ans à l'étranger, et ceux qui possèdent une expérience familiale de l'international du fait de la profession de leurs parents.

31 Il est certes difficile de distinguer un par un les héritiers du reste des contacts. Mais, à partir des données relatives à la profession des pères, on peut dégager des nuages de contacts n'appartenant pas à la grande bourgeoisie turque. Ainsi, il apparaît clairement qu'une grande partie des contacts n'ayant pas vécu hors de leur pays d'origine ne correspondent pas à la définition des héritiers. Il s'agit d'une part des relations de travail activées après leur retour par Murat, Gaye, Selin et Bilge (graphes nº 3.1 et 3.2, nuages "travail retour»). Ces quatre enquêtés ont intégré à leur retour différents milieux professionnels (publicité, journalisme et photographie) où ils ont côtoyé des individus issus pour la plupart de milieux sociaux différents du leur. Dès lors, si l'on 
écarte les relations non-électives activées dans le cadre professionnel, l'homogénéité sociale des réseaux est suffisamment forte pour que l'on puisse parler de trajectoires migratoires spécifiques aux héritiers. Il s'agit d'autre part de contacts de nationalité étrangère: les relations de travail de nationalité américaine de Kaan à New York (graphe $\mathrm{n}^{\circ} 3.2$, nuage "New York travail»), les amis d'université anglais de Bilge à Londres (graphes $n^{\circ} 3.2$, nuage "Londres - université »), ou encore les DJ de musique électronique européens et américains avec lesquels Bulut se produit sur scène (graphe $\mathrm{n}^{\circ}$ 3.3, nuage « réseau DJs Europe - NY »). Il s'agit enfin de nuages de contacts dont l'âge ne correspond pas, tels que les grands-parents, oncles et tantes de la famille élargie de Kaan (graphe $n^{\circ} 3.2$, nuage "famille»). On peut ainsi parler d'une cohorte d'héritiers migrants, $84 \%$ (794/948) des contacts de nationalité turque étaient âgés de 23 à 33 ans au moment de l'enquête. $65 \%(515 / 794)$ d'entre eux ont vécu au minimum un an à l'étranger, dont $82 \%$ au moins trois ans (plus encore si l'on considère que près d'un tiers de l'échantillon considéré se trouvait toujours à l'étranger).

Or, s'il est logique que les contacts rencontrés à l'étranger y aient vécu plusieurs années, on retrouve également de nombreux contacts ayant une longue expérience de l'international dans les nuages de liens activés durant l'enfance. Ces dynamiques de réseaux renvoient aux distinctions entre milieux bourgeois évoquées en introduction. Dans les réseaux des enfants de diplomates et de hauts fonctionnaires internationaux, en particulier ceux de Nesli et Bilge (graphe $\mathrm{n}^{\circ}$ 3.2), on constate une sédimentation des maillages hérités, spécifiques des familles circulantes. La notion de sédimentation traduit l'idée que les "amis de la famille» et les "amis des amis de la famille", dont les parcours migratoires sont similaires à ceux de Nesli, ont résisté mieux que les autres maillages à l'épreuve du temps. Rencontrés pour la plupart durant l'enfance, ils sont restés bon gré mal gré au centre du réseau, c'est-à-dire en interconnexion avec des individus rencontrés à toutes les étapes du parcours d'Ego. Dans les réseaux des enquêtés issus familles cosmopolites, notamment Kaan et Gaye (graphes nº 3.1 et 3.2), dont les parents ont également fait leurs études à l'étranger, les contacts les plus connectés ont été rencontrés dans le cadre scolaire (nuages "primaire ", «lycée ", " amis d'amis du lycée »), mais ils ont été systématiquement introduits aux parents, qui occupent une position centrale dans le réseau. Enfin, dans les réseaux de Murat et Eda (graphes $\mathrm{n}^{\circ} 3.1$ et 3.3), dont la socialisation internationale constitue une forme de capital scolaire nouvelle pour leur famille, les "amis d'enfance " issus d'un même ancrage microlocal (les quartiers chics de la rive asiatique d'Istanbul) ont évolué en un cercle de contacts forts distributeurs participant au tissage des cliques à chaque étape du parcours d'Ego. Leur apprentissage de la culture internationale, rendu nécessaire par l'évolution des critères du prestige relatifs à l'expérience de l'étranger, s'est fait sans l'intervention des familles, mais sans déracinement.

\section{Des départs précoces}

33 L'avancement généralisé du moment des départs à l'étranger des étudiants turcs repose sur des dynamiques nationales renvoyant à l'état du système d'enseignement et à l'opinion qu'en ont les familles. Sur le tableau $\mathrm{n}^{\circ} 6$, on observe d'importantes variations entre les dix principales communautés étudiantes étrangères aux États-Unis. Tandis que la majorité des étudiants en provenance de l'Inde (73\%), de la Chine (80\%) et de la Thaillande (63\%) viennent suivre un cursus universitaire long ( 4 ans en moyenne), les étudiants en provenance du Japon (69\%), de l'Indonésie (69\%) et, dans une moindre 
mesure, du Mexique (57\%) et du Canada (53\%), ne viennent fréquemment suivre qu'une formation de 3e cycle ( 1 ou 2 ans pour les Masters et MBA; 4 ans en moyenne pour les $\mathrm{Ph}$. D). Ces différences sont liées d'une part à des facteurs économiques. En effet, une partie de ces étudiants migrants ne peut se permettre de financer un cursus universitaire complet, d'autant qu'il est beaucoup plus difficile d'obtenir une bourse d'étude pour un 1er cycle universitaire que pour un mastère ou un doctorat. Quant aux MBA, ils sont fréquemment financés par les entreprises, qui envoient leurs cadres en complément de formation. Elles sont d'autre part liées à la nature des systèmes éducatifs de ces pays et à l'évolution des critères du prestige académique. Dans les pays possédant un système universitaire performant au regard des critères internationaux, tels que le Japon ou le Canada, qui sont par ailleurs des pays d'immigration étudiante, l'acquisition d'un troisième cycle aux États-Unis permet de se distinguer du reste des étudiants nationaux. 9 des 10 MBA les plus cotés se trouvent en effet aux États-Unis. Seul l'Insead, dont le cursus se déroule pour moitiés à Paris et à Singapour, trouble l'hégémonie des universités américaines (Financial Times, 2006). Il faut enfin prendre en compte les intentions de retour de ces populations étudiantes migrantes. Alors qu'une majorité d'étudiants indiens et chinois projettent de rester aux États-Unis après la fin de leur cursus, les étudiants japonais ou indonésiens viennent obtenir une formation de complément appréciée par les entreprises de leur pays (Tremblay, 2002). En suivant un cursus complet dans une université américaine, les étudiants indiens ou chinois n'ayant pas l'intention de rentrer dans leur pays ont d'autant plus de chances de trouver une entreprise disposée à leur financer un visa de travail une fois la période de practical training terminée.

Au regard de ces trois facteurs, la Turquie présente un statut bigarré. $57 \%$ de l'ensemble des étudiants turcs viennent aux États-Unis pour débuter leurs études supérieures, tandis que $34 \%$ d'entre eux y poursuivent un $3^{\mathrm{e}}$ cycle et $9 \%$ une formation linguistique de complément ( $\mathrm{cf}$. tableau $\mathrm{n}^{\circ} 6$ ). Cela contraste avec les pourcentages tirés des réseaux des huit enquêtés, où la majorité des étudiants viennent suivre une formation complète, mais ne souhaitent pas faire carrière à l'étranger. Au sein de la grande bourgeoisie turque, l'acquisition d'un troisième cycle est devenue progressivement synonyme de diplôme international. 95 \% des contacts ayant obtenu un diplôme de troisième cycle ont opté pour une université étrangère. Néanmoins, seuls $22 \%$ de l'ensemble contacts de nationalité turque ont suivi un troisième cycle. En outre, le diplôme de troisième cycle n'est le plus souvent qu'une étape dans le cycle migratoire des héritiers. Il s'inscrit dans un projet de formation internationale complet. Ainsi, seuls $7 \%$ des contacts de nationalité turque ayant vécu à l'étranger sont partis pour suivre un troisième cycle après un cursus universitaire en Turquie. Autrement dit, la précocité des départs ne se traduit pas par une précocité des retours. Au contraire, elle pousse les héritiers à prolonger leur formation internationale, d'autant que les MBA les plus sélectifs exigent une expérience professionnelle minimum de deux ans.

Tableau 6 : Les étudiants étrangers aux Etats-Unis par niveau universitaire et pays d'origine

\begin{tabular}{|l|l|l|l|l|}
\hline Classement & Pays d'origine & $\mathbf{3}^{\mathbf{e}}$ cycle \% & 1er et 2nd cycles \% & Formations Linguistiques \% \\
\hline 1 & Inde & 22 & 73 & 5 \\
\hline 2 & Chine & 14 & 80 & 6 \\
\hline
\end{tabular}




\begin{tabular}{|l|l|l|l|l|}
\hline 3 & Corée du Sud & 41 & 47 & 12 \\
\hline 4 & Japon & 69 & 19 & 12 \\
\hline 5 & Taiwan & 37 & 53 & 10 \\
\hline 6 & Canada & 53 & 42 & 5 \\
\hline 7 & Mexique & 57 & 36 & 7 \\
\hline 8 & Turquie & 34 & 57 & 9 \\
\hline 9 & Indonésie & 69 & 25 & 6 \\
\hline 10 & Thailande & 28 & 63 & 9 \\
\hline & Total mondial & 47 & 45 & 7 \\
\hline
\end{tabular}

Source : Institute of International Education, Open Doors 2001.

\section{Faire ses preuves}

Beaucoup parmi les contacts des huit réseaux ayant étudié aux États-Unis ont profité des quatre ans qu'ils ont passés à étudier pour rester quelques années de plus à travailler, ce qui a compliqué d'autant la programmation de leur retour. Le visa accordé aux étudiants étrangers (F-1) prévoit depuis 1982 (régulation fédérale 8 CFR 214.2 f.10), 12 mois d'optional practical training dans un secteur d'activité directement lié à la discipline dans laquelle le diplôme a été obtenu, sachant que le cursus suivi doit avoir une durée minimale de 9 mois et que les étudiants ayant obtenu un certificat linguistique sont inéligibles (Shusterman, 2005). De plus en plus de jeunes diplômés étrangers mettent ainsi à profit ce droit d'entrée dans le marché du travail américain pour acquérir une première expérience professionnelle. Néanmoins, le système américain est construit de manière à ce que les immigrants soient constamment dans l'attente d'un nouveau visa de travail. Une fois la période de practical training écoulée, le problème du visa de travail se pose à nouveau: il faut rentrer en Turquie ou bien obtenir d'une entreprise qu'elle fasse les démarches nécessaires pour obtenir un visa de travail temporaire $\mathrm{H}-1 \mathrm{~B}$ de trois ans renouvelable une fois16, et éventuellement, à terme, un permis de travail permanent (green card).

En outre, pour les garçons, se pose également la question du service militaire, qu'il est possible de réduire à un mois au lieu de quinze, si l'on a travaillé au moins trois ans à l'étranger. Kaan et Murat, qui possèdent une telle expérience professionnelle internationale, ont ainsi fait leur service militaire immédiatement à leur retour de l'étranger sans être mélangés au reste des appelés. Cela explique qu'ils aient tous deux noué des liens d'amitié durables avec certains retournants de leur contingent (cf. graphe $\mathrm{n}^{\circ} 3.2$, nuage « service militaire " pour Kaan), contrairement à Bulut, qui a fait un service militaire de 5 mois (au lieu de 15 mois en tant que diplômé de l'université) dans un contingent régulier. 

postuniversitaires à l'étranger s'est répercuté sur les filles. Larry Turns (premier conseiller d'orientation spécialisé sur les études aux États-Unis, employé à plein-temps par le Lycée Koç depuis 1994) explique ainsi que la principale évolution des dix dernières années est l'allongement de la durée de la migration ${ }^{17}$. Alors qu'auparavant la majorité de ces jeunes partaient uniquement pour des troisièmes cycles, ils partent aujourd'hui dès la fin du lycée, voire à la fin du primaire, comme les 21 contacts diplômés des pensions de Suisse Romande (cf. graphe $\mathrm{n}^{\circ}$ 2.1). Ils restent ensuite plusieurs années afin d'acquérir une première expérience professionnelle. De plus en plus d'étudiantes migrantes choisissent de prolonger leur séjour à l'étranger après la fin de leurs études. À leur retour en Turquie, ces jeunes filles évitent ainsi d'être surclassées dans le marché du travail par les garçons possédant une telle expérience professionnelle. Les huit réseaux égocentrés affichent ainsi une parité totale : $66 \%$ (246/374) des jeunes filles et $66 \%$ (269/420) des garçons de nationalité turque âgés de 23 à 33 ans ont vécu au moins un an à l'étranger ; $61 \%(150 / 246)$ de ces jeunes filles et $61 \%(164 / 269)$ de ces garçons ont vécu plus de 4 ans à l'étranger. d'accéder à des postes dont le statut est reconnu en Turquie. Ces objectifs conviennent bien aux cabinets de consulting et d'expertise financière, qui proposent des salaires élevés à de jeunes diplômés, sans intention de les garder plus de quelques années. Des firmes de renommée internationale telles que McKinsey, Boston Consulting et Bain, en tête $\mathrm{du}$ classement 2005 des cabinets de conseil les plus prestigieux (Vault, 2005a), ou Goldman Sachs, Morgan Stanley et Blackstone, en tête du classement des cabinets d'expertise financière (Vault, 2005b) s'inscrivent dans une relation spécifique d'échange avec les jeunes élites migrantes, qu'elles recrutent à la sortie des universités américaines les plus cotées. Disposées à investir des sommes importantes (salaires et visas) dans de jeunes recrues prêtes à travailler sans réserve, elles leur offrent en échange leur nom (reconnu en Turquie), ainsi que la possibilité de faire leurs preuves dans un marché compétitif. Le développement et la structure des cabinets de conseil et d'expertise en France ont été analysés par Odile Henry (Henry, 1992). Ce secteur, en perpétuelle expansion depuis le début des années 1980, est décrit comme un espace de positions nouvelles aux limites incertaines, employant aussi bien des jeunes diplômés que des professionnels expérimentés, issus de formations et de secteurs d'activités extrêmement hétérogènes (droit, économie, gestion, sociologie, etc.). En même temps, Henry évoque l'existence d'une compétence de classe héritée, particulière à ce secteur professionnel, observant que «l'aisance sociale dont il faut disposer semble croître à mesure qu'on s'élève dans la hiérarchie des cabinets de conseil ». De ce point de vue, ce secteur est donc particulièrement favorable aux héritiers migrants. En même temps, l'entrée dans un cabinet de conseil n'est qu'une étape permettant d'accumuler de l'expérience et du prestige avant de s'inscrire dans un MBA ou de rentrer dans leur pays. Les cabinets de conseil, pour leur part, gagnent à recruter de plus en plus de jeunes diplômés, qu'ils peuvent "vendre » moins cher que les consultants confirmés, afin de satisfaire à leurs exigences de croissance.

À la sortie de l'université, ces héritiers ont cherché logiquement à occuper ce que Joseph Epstein décrit comme «les postes actuellement les plus admirés aux ÉtatsUnis », c'est-à-dire «l'homme ou la femme qui ne procure pas le service ou le produit mais qui l'amène sur le marché, en acquérant au passage un important profit ». Ces 
hommes et ces femmes, poursuit Epstein, sont «agent, broker, trader, investment banker, operator...» (Epstein, 2002: 44). On retrouve ainsi ces mêmes professions chez une partie importante des contacts de nationalité turque âgés de 23 à 33 ans qui se trouvaient encore à l'étranger au moment de la construction des réseaux. Tandis qu'un quart d'entre eux (45/179) y poursuivaient encore des études (dans la majorité des cas un troisième cycle), plus de la moitié des professionnels (70/134, soit $52 \%$ ) travaillaient dans la banque, dans un cabinet de conseil, ou dans une multinationale en tant que manager, expert en marketing ou comptable. Quant à l'autre moitié (64/134), il s'agit d'individus qui exerçaient pour beaucoup dans de gros cabinets d'avocats (15 liens) et d'architectes (5 liens), ou dans de grandes entreprises de télécommunication en tant qu'ingénieurs (8 liens), informaticiens ( 9 contacts) ou publicistes (6 contacts). Enfin, 5 contacts géraient une filiale étrangère de l'entreprise familiale. Six des huit enquêtés ont ainsi accepté de travailler dans des multinationales à l'étranger plusieurs années, certes en échange de salaires conséquents, mais dans des conditions de travail qu'ils semblent ne pas être prêts à accepter en Turquie, au moment de leur retour.

\section{Bourgeoisie retournante}

La migration et le retour des héritiers participent d'un régime temporel particulier. Leurs trajectoires migratoires, dont on a vu qu'elles reposent sur des temporalités précises, élargissent le champ des possibles de cette cohorte d'héritiers. Le retour se traduit par une conjonction des temporalités ouvrant sur une "période-critique " (Bidart, 2006), durant laquelle ces néoretournants éprouvent le besoin de mettre en cohérence leur parcours. Elle réside dans le désir paradoxal de certains retournants de refuser la "voie facile ", c'est-à-dire l'entreprise familiale (la carrière diplomatique pour les enfants de diplomates), le réseau des parents, la maison des parents, etc.. Bien que prévue de longue date, la situation du retour entraîne une rupture dans la continuité des enchaînements. La décision du retour intervient à un carrefour biographique, qui repose sur une pluralité de choix et d'enjeux : opportunités professionnelles à la sortie de l'université, questions liées au visa et possibilités d'obtenir une green card, événements liés à la conjoncture économique en Turquie et à l'étranger, dépendances liées au service militaire pour les garçons, rencontre amoureuse, etc.. Cette décision est ainsi parfois prise en décalage avec le projet familial établit avant le départ. Ainsi, le moment du retour constitue une rupture dans la trajectoire planifiée et intégrée (la réussite scolaire est une nécessité interne, personnelle) et permet l'émergence de nouvelles appétences. En même temps, la synchronie des trajectoires de nombreux contacts au sein d'un réseau de sociabilité permet l'activation d'efficaces ressorts communs d'action propres à ce groupe et qui se développent lors du retour.

\section{Les théories des migrations du retour}

41 La situation du retour est désormais un seuil important dans le cycle de vie de la nouvelle génération d'héritiers de la grande bourgeoisie turque. Les théories des "return migrations» permettent de décrire les origines de sa généralisation et ses dynamiques internes. Les théories de la migration du retour (e.g. motivations, rôles, ressources, préparation du retour) ont été compilées et discutées par Jean-Pierre Cassarino (Cassarino, 2004). Cassarino passe en revue les cinq grandes approches théoriques: l'analyse néo-classique, celle relevant de la «nouvelle économie des 
migrations de travailleurs ", l'approche structurelle, transnationale, et enfin l'analyse des réseaux sociaux. Le débat scientifique sur la situation du retour a réellement débuté dans les années 1980, suite aux publications de sociologues tels que Francesco Cerase (Cerase, 1974) et d'anthropologues tels que George Gmelch (Gmelch, 1980), qui furent les premiers à s'intéresser non seulement aux motivations des retournants mais aussi à leurs attentes. Tandis que les trois premières approches tendent à réduire le cycle migratoire à l'histoire d'un échec ou d'un succès, l'approche transnationale et l'application de la théorie des réseaux sociaux à la migration du retour partent de l'hypothèse que, sous certaines conditions, les retournants peuvent jouer un rôle central dans leur société d'origine ${ }^{18}$. Les travaux relevant de la théorie des réseaux sociaux, en particulier, proposent de s'intéresser aux solidarités fondées non seulement sur des attributs communs, mais aussi et surtout des intérêts communs. Le succès des projets et des initiatives des retournants est lié à la maitrise de l'utilisation des réseaux sociaux et économiques transfrontaliers, mais aussi au capital social de départ des migrants et à l'expérience passée de l'international des familles. Ainsi, les motivations expliquant la décision du retour définitif tiennent autant aux opportunités offertes par le pays d'origine qu'aux ressources personnelles. Cette nouvelle approche des migrations internationales fait du retour non plus la dernière mais la première étape vers l'achèvement du projet migratoire. À partir de ce cadre d'analyse, il est possible de redéfinir la notion de retournant. Cassarino avance ainsi quatre raisons d'effectuer un tel travail : la diversification des flux migratoires oblige à ne plus considérer que les migrations ne concernent que les "travailleurs » («labour migrants»), qui suppose de créer de nouvelles catégories telle que celle d'«étudiant migrant»; l'ouverture à l'économie de marché et le développement du secteur privé dans de nombreux pays en voie de développement - notamment en Turquie - ont significativement accru les possibilités de reconversion des ressources acquises à l'étranger par les retournants ; la diminution des coûts des transports internationaux a fait du retour un processus en plusieurs étapes; le développement récent de nouvelles technologies permettant une meilleure circulation des flux d'informations (Internet, téléphone portable, etc.) permet aux futurs retournants de mieux se préparer (Cassarino, 2004).

$\mathrm{Au}$ final, le déroulement du retour est largement influencé par les motivations initiales de la migration. L'étude des réseaux de sociabilité des retournants montre l'importance des relations interpersonnelles et des ressources familiales dans la préparation du retour. Il est possible de définir les critères régissant ce que Cassarino appelle le « degré de préparativité des futurs retournants" ("prospective returnees' preparedness»). L'évaluation du degré de préparativité repose sur l'idée que le retour ne constitue pas la fin du cycle migratoire, mais qu'il s'intègre dans un système circulaire de relations sociales et économiques et d'échanges, qui facilite la réintégration en convoyant informations, connaissances et marqueurs d'appartenance. Un haut degré de préparativité requiert dès lors non seulement la volonté du migrant, mais aussi la mobilisation de ressources et d'informations sur les conditions de son retour. En outre, le degré de préparativité n'est pas seulement tributaire des ressources accumulées à l'étranger, mais également, en amont, du capital social de départ (essentiellement procuré par la famille), et, en aval, de la perception que le futur retournant a des changements institutionnels, économiques et politiques intervenus en Turquie durant son absence. 


\section{Héritiers du retour}

43 Au sein de la grande bourgeoisie turque, la migration des héritiers n'est pas un but en soi, mais elle s'est imposée comme une étape nécessaire. Il s'inscrit dans un projet familial s'accomplissant à terme par l'institution de l'héritier. Le retour étant projeté en même temps que le départ, l'équation de leur migration présente une inconnue de moins que celle du reste des migrants. Quant au profil de ces retournants, il satisfait toutes les conditions établies comme étant nécessaires au succès du retour :

- le capital social de départ des héritiers est plus élevé que celui du reste des migrants. Durant les années de migration, la construction des «identités transnationales » est facilitée par l'assurance procurée par le statut social de l'héritier dans son pays d'origine. Les ressources économiques familiales ont également une importance, en particulier pour les héritiers turcs, le temps des études étant entièrement financé par les familles. Cela n'est pas le cas dans une partie des familles bourgeoises européennes ou américaines, qui considèrent que les héritiers doivent financer en partie par eux-mêmes leur voyage initiatique, les «petits boulots » ou « boulots d'été » participant de l'apprentissage ;

- en corrélation avec ce premier élément, la fréquence des retours périodiques des héritiers est supérieure à celle du reste des migrants. Ils donnent l'occasion, outre de conserver des liens étroits avec la famille, de retrouver les amis d'enfance (rencontrés au cours du cursus scolaire ou au travers de la famille) partis pour des destinations différentes, et de tisser des liens avec d'autres héritiers migrants ;

- l'expérience de l'international des parents, oncles et tantes, voire des grands-parents d'une partie des héritiers turcs sert au perfectionnement de leurs trajectoires migratoires, tant en termes de durée que de préparativité au retour. Par ailleurs, les contacts entretenus par les familles bourgeoises à l'étranger permettent de préserver l'entre-soi durant les années à l'étranger ;

- les « réseaux sociaux et économiques transfrontaliers », bien qu'inutiles à la préparation du retour des héritiers du fait de l'importance du capital de départ, sont cependant mobilisés après le retour. En particulier, la possibilité que les héritiers ont de garder un «pied à terre " après leur retour dans les lieux de leur migration leur permet d'ajouter à leur «double identité » une «double adresse », constituant une prime de statut pour leurs investissements ;

- la fréquence des retours périodiques et l'utilisation professionnelle des réseaux transfrontaliers font du retour un processus en plusieurs étapes, augmentant le degré de préparativité au moment du retour définitif.

- les succès académiques et professionnels des héritiers migrants sont célébrés dans les médias turcs, tant à l'étranger qu'à leur retour.

Néanmoins, parce qu'ils partent avec un avantage, les attentes et les appétences des héritiers sont également supérieures, au moment du retour, à celles du reste des retournants. Forts de l'accomplissement d'un cycle migratoire «idéal ", ils ne s'en trouvent pas moins insatisfaits des opportunités qui leurs sont offertes. Ainsi, l'entrée dans l'entreprise familiale est jugée par une partie des héritiers retournants de la bourgeoisie d'affaires comme une "voie facile", et, tant les salaires que les profils d'emplois proposés par les entreprises turques, sont vécus comme une régression. Reste que ces inadéquations correspondent aux symptômes de la situation de rupture engagée par la décision du retour définitif. En partant de l'idée selon laquelle le retour n'est que la première étape vers l'achèvement du projet migratoire, il est possible de mettre en évidence un cycle du retour des héritiers. Ainsi, leur institution n'intervient pas 
à leur retour mais à l'issue d'un processus de réadaptation, dont il faut déterminer non seulement la durée, mais aussi les conséquences sur le fonctionnement des mécanismes de la reproduction sociale en grande bourgeoisie turque.

\section{Une émancipation à retardement}

Le retour marque en principe la fin de la formation des héritiers, de leur initiation, mais le statut du retournant offre un espace de liberté les poussant à prolonger cette période d'expérimentation non-planifiée. Le retour en Turquie, dont le moment même constitue déjà pour certains une première rupture dans la planification du voyage initiatique, ne correspond pas à une stabilisation des attentes, des envies, mais au contraire à une amplification des questionnements individuels. Autrement dit, ce n'est pas durant la période passée à l'étranger que la remise en question de soi est la plus forte, mais au moment du retour. Les conditions du couronnement génèrent dès lors un équilibre instable. L'effacement de la distance physique avec le milieu d'origine autorise paradoxalement une prise de distance vis-à-vis de celui-ci. Les décalages et les désajustements liés à la situation du retour correspondent à la réactivation de schèmes d'action incorporés qui se déclenchent une seconde fois en sens inverse de la première (durant la socialisation à l'étranger). Cet effet boomerang se traduit éventuellement par des retournements et des renversements dans la trajectoire planifiée.

La situation du retour engage un processus décisionnel correspondant au franchissement des seuils de la jeunesse (la fin des études et l'élaboration de projets professionnels à long terme). Elle constitue donc à la fois une rupture biographique et le bouleversement de l'organisation du quotidien, ce qui facilite doublement la reformulation des schèmes qui constituent les habitudes. En même temps, la situation du retour a cela de particulier que le sujet est pris entre deux dynamiques individuelles. D'une part, la volonté de retrouver certaines des anciennes habitudes : celles d'avant son départ, et celles qu'il a intériorisées durant les années à l'étranger, qu'il tente, cette fois consciemment, de transposer à sa nouvelle vie stambouliote. De l'autre, la volonté de créer un nouvel enchaînement introduisant une interaction ouverte. La mise en cohérence des habitudes passe ainsi par la reconfiguration du système des relations, dès lors que l'étendue du champ des possibles d'un individu est subordonnée à sa plus ou moins grande capacité à agir sur le réseau d'interdépendances dans lequel il est inscrit. À ce moment particulier de leur existence, les habitudes seules ne sont plus en mesure de répondre aux questions qu'ils se posent. En effet, les schèmes incorporés sont en contradiction avec le processus d'autonomisation et d'expérimentation, qui s'accélère lors du retour. D'une manière ou d'une autre, le voyage initiatique doit être justifié. Cela se traduit par le développement de la pensée analytique, c'est-à-dire par une plus grande réflexivité, la réflexivité étant le stade préalable à la véritable incorporation des schèmes intériorisés durant la socialisation internationale.

La similarité des parcours migratoire et la synchronie des retours permettent, au moment du retour, une autonomisation temporelle, née de « temporalités vécues ayant suffisamment de consistance pour déboucher et se cristalliser sur de véritables modes de vie » (Schehr, 1999). Mais cette autonomisation intervient à contretemps. Le retour ne joue pas comme prévu son rôle de rite d'institution. Il fait intervenir au contraire des effets de résonance - c'est-à-dire des vibrations liées à la mise en adéquation de schèmes dissonants ${ }^{19}$ - difficilement maîtrisables. Cette période d'adaptation est un temps de 
sociabilité intense, qui se traduit à la fois par un sentiment de grande liberté (le prestigieux "diplôme d'une université étrangère » est un visa d'entrée dans le monde du travail), et par la volonté de profiter d'un temps d'expérimentation limité (il faudra tôt ou tard débuter une carrière, que ce soit dans l'entreprise familiale ou non, et choisir son futur conjoint). Néanmoins, les effets de résonance observés chez la plupart des enquêtés ne se sont en aucune façon liés à des effets de déclassement, comme cela peut être le cas par exemple pour des expatriés ayant bénéficié à l'étranger de salaires et de privilèges auxquels ils doivent renoncer une fois rentrés dans leur pays. En outre, un nouveau déménagement ne devrait pas poser problème pour ces jeunes professionnels habitués à devoir s'adapter, seuls ou en famille, à de nouvelles villes et à de nouvelles cultures. Or, c'est précisément cela qui pose problème, car il est paradoxalement plus facile de s'adapter à un nouveau milieu que de se réadapter à son milieu d'origine. En effet, la réactivation des schémas d'expérience mis en veille durant la migration suppose de renoncer aux distinctions conférées par les habitudes migrantes, dont l'entretien aide par ailleurs à justifier le rôle d'héritier retournant dans la société turque.

En conclusion, la dimension migratoire internationale des rites d'institution en milieu bourgeois est désormais l'élément central d'un modèle de transmission déterminant l'accès aux formations d'excellence et aux positions les plus sélectives - tant dans le champ international que dans le milieu d'origine. La singularité du cycle migratoire de la cohorte d'héritiers présentée est liée à l'imbrication de pendularismes longs (la durée totale de la migration) et de pendularismes courts (les cycles quadriennaux des familles de diplomates et, surtout, les retours estivaux). Cette migration sans déracinement, renforcée par la concentration des destinations, permet à la fois une socialisation internationale réussie, à savoir l'incorporation d'habitudes telles que le contrôle de soi, la distanciation et le recul par rapport à soi-même (Le Wita, 1988 : 161), et l'entretien des valeurs transmises dans le monde domestique. La formation de ces héritiers satisfait donc aux exigences de la transmission et de l'accumulation des capitaux. La mesure, la sobriété, la modestie et la simplicité viennent contrebalancer l'aisance, l'assurance et la revendication de l'excellence. Par suite, les difficultés rencontrées au moment du retour renvoient à la difficile adéquation de l'héritage et de l'innovation. Bien qu'elle consiste avant tout à tirer la « leçon de l'économie familiale » (Gotman, 1988: 12), l'action d'hériter est compatible avec l'innovation en tant qu'introduction d'une nouveauté dans l'usage. Toutefois, l'institution de l'héritier nécessite obligatoirement un compromis entre l'indépendance au quotidien et la réintégration dans les hiérarchies propres au monde domestique. Le cycle migratoire des héritiers se clôt finalement avec la fin du retour, qui marque le retour à l'équilibre et la résolution des contradictions par l'incorporation des habitudes migrantes. Cela ne signifie pas que ceux des retournants qui avaient bifurqué reviennent à la trajectoire planifiée. Au contraire, la bifurcation est raccordée au régime de succession, dont les habitudes migrantes ont transformé les conditions.

Murat : 


\section{BIBLIOGRAPHIE}

ABADAN-UNAT Nermin (1996) The Socio-Economical Aspects of return Migration to Turkey, in Robin Cohen Ed., Sociology of Migration, Cheltenham UK, Brookfield US, E. Elgar Publishing, pp. 29-59.

BATES Donald la Vere (1973) The Origin and Career Path Development of the Modern Turkish Business Elite, Thèse de doctorat non publiée, Université de l'Arkansas.

BIDART Claire (2006) Crises, decisions et temporalities : autour des bifurcations biographiques, Cahiers Internationaux de Sociologie, à paraître, Vol. 120.

BLUMER Herbert (1969) Fashion : from Class Differenciation to Collective Selection, Sociological Quaterly (10), pp. 275-291.

BOURDIEU Pierre, BOLTANSKI Luc, SAINT MARTIN Monique de (1973), Les stratégies de reconversion. Les classes sociales et le système d'enseignement, Information sur les sciences sociales, XII (5), pp. 61-113.

BUĞRA Ayşe (1994) State and Business in Modern Turkey. A Comparative Study, Albany, State University of New York Press.

CASSARINO Jean-Pierre (2004) Theorising Return Migration : the Conceptual Approach to Return Migrants Revisited, International Journal on Multicultural Societies, 6 (2), pp. 253-279.

CERASE Francesco P. (2004) Expectations and Reality : a Case Study of Return Migration from the United States to Southern Italy, International Migration Review, 8 (2), pp. 245-262.

De NOOY Wouter, MRVAR Andrej, BATAGELJ Vladimir (2005) Exploratory Social Network Analysis with Pajek, Cambridge University Press, NY.

EPSTEIN Joseph (2002) Snobbery, Boston, Houghton Mifflin Co.

ERNER Guillaume (2004) Victimes de la mode? Comment on la crée? Pourquoi on la suit? Paris, la Découverte.

FAGUER Jean-Pierre (1991) Les effets d'une éducation totale. Un collège jésuite, 1960, Actes de la Recherche en Sciences Sociales, 86-87, pp. 25-43.

Financial Times (2006) Top 100 Global MBA Programmes, URL :

http://news.ft.com/businesslife/mba

FINDLEY Carter V. (1989) Ottoman Civil Officialdom : a Social History, Princeton University Press.

GENÇLER Ayhan, ÇOLAK Aytül (2003) Türkiye'den Yurtdışına Beyin Göçü : Ekonomik ve Sosyal Etkileri, communication présentée au 1. Bilgi, Ekonomi ve Yönetim Kongresi [L'exode des cerveaux de la Turquie vers l'étranger : impacts économiques et sociaux, communication présentée au $1^{\mathrm{er}}$ Congrès sur la Connaissance, l'Économie et la Gouvernance].

GLASER William A., HABERS G. Christopher (1974) The Migration and Return of Professionals, International Migration Review, 8 (2), pp. 227-244.

GMELCH George (1980) Return Migration, Annual Review of Anthropology, 9, pp. 135-159.

GOTMAN Anne (1988) Hériter, Paris, PUF. 
GÖÇEK Fatma Müge (1999) Burjuvazinin Yükselişi ve İmparatorluğun Çöküşü [L'Ascension de la bourgeoisie et l'effondrement de l'Empire], Ankara, Aytaç.

GÖLE Nilüfer (1986) Mühendisler ve Ideoloji, Istanbul, Metis.

GRIBAUDI Maurizio Ed. (1988) Espaces, temporalités, stratifications. Exercices sur les réseaux sociaux, Paris, École des Hautes Études en Sciences Sociales.

HENRY Odile (1992) Entre savoir et pouvoir. Les professionnels de l'expertise et du conseil, Actes de la Recherche en Sciences Sociales, 95, pp. 37-55.

LE WITA Beatrix (1988) Ni vue, ni connue. Approche ethnographique de la culture bourgeoise, Paris, Maison des Sciences de l'Homme.

MANÇO Ural (2004) Turcs d'Europe : une présence diasporique unique par son poids démographique et sa complexité sociale, in Ural Manço Ed., Turquie : vers de nouveaux horizons migratoires ?, Paris, Turin, Budapest, L'Harmattan, coll. Compétences interculturelles, pp. 153-178. PINÇON Michel et PINÇON-CHARLOT Monique (2000) Sociologie de la bourgeoisie, Paris, La Découverte, coll. Repères.

ROBINSON Dwight (1961) The Economics of Fashion Demand, Quaterly Journal of Economics, 75 (3), pp. 358-386.

SAINT MARTIN Monique de (1993) L'Espace de la noblesse, Paris, Métallié, coll. Leçons de choses. SCHEHR Sébastien (1999) La conquête de l'autonomie temporelle, Temporalistes, 40, pp. 16-25. SHUSTERMAN Carl (2005) Temporary Work Visas for Foreign Students, URL : http://www.latpro.com/USER/articles/Visa2.php?Lang=PORT

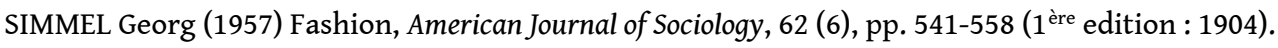
SORAL Erdoğan (1974) Özel Kesimde Türk Müteşebbisleri [Les entrepreneurs turcs dans le secteur privé], Ankara, İktisâdî ve Ticarî ìlimler.

TAPIA Stéphane de (1996) La multipolarisation de la migration turque, in Emmanuel Ma Mung Dir., Mobilités et investissements des émigrés : Maroc, Tunisie, Turquie, Sénégal, Paris, Montréal, L'Harmattan, pp. 33-47.

TREMBLAY Karine (2002) Student Mobility Between and Towards OECD Countries : A Comparative Analysis, International Mobility of the Highly Skilled, Paris, OECD Proceedings, pp. 39-67.

UNESCO (2005) Recueil de données mondiales sur l'éducation 2005, Montréal, Institut de Statistique de l'UNESCO.

US News World Report (2005), URL :

www.usnews.com/usnews/edu/college/rankings/rankindex brief.php

VAULT (2005a) Vault Top 50 Consulting Firms 2005. URL : www.thevault.com

VAULT (2005b) Vault Top 50 Banking Employers 2005. URL : www.thevault.com

VEBLEN Thornstein (1934) The Theory of Leisure Class, NY, The Modern Library (1 ère édition, 1899). WAGNER Anne-Catherine (2001) Les nouvelles élites de la mondialisation, une immigration dorée en France, Paris, PUF. 


\section{ANNEXES}

\section{Présentation des enquêtés}

Murat est né en 1975 à Istanbul. Il a une sœur aînée. Son père possède et dirige une entreprise d'import-export dans le secteur de la chimie. Après des études secondaires à Istanbul, Murat part pour Boston, dans l'une des nombreuses universités privées de la ville. Il travaille ensuite deux années à New York dans l'investissement bancaire, puis deux années de plus à Londres. Il décide de rentrer à Istanbul début 2001, soit un an avant le début de l'enquête. Murat travaille depuis lors pour une grande entreprise anglaise de publicité.

\section{Gaye :}

Gaye est née en 1979 à Izmir, mais sa famille est stambouliote. Elle a une sœur aînée. Son père a débuté en tant qu'universitaire, puis a fait carrière en tant que cadre dirigeant dans le secteur des medias, avant de fonder récemment sa propre entreprise dans le secteur de la télécommunication par satellite. Née à Izmir, Gaye a vécu les premières années de sa vie à Hawaï. Elle a ensuite suivi ses études primaires et secondaires à Istanbul, avant de partir à Boston pour des études de journalisme et communication. Une fois diplômée, elle décide de rentrer en Turquie pour débuter, sans attendre, une carrière dans le journalisme. Bien qu'étant la plus jeune des enquêtés, Gaye est donc aussi celle qui est rentrée le plus tôt, début 2000, soit deux ans et demi avant le début de l'enquête.

\section{Eda :}

Eda est née en 1975 à Istanbul. Elle a un frère cadet. Son père possède et dirige une entreprise d'architecture en bâtiment. Sa mère, qui est, avec la mère de Kaan, la seule parmi les mères des huit enquêtés à exercer une activité professionnelle, est architecte d'intérieur. Eda, après des études primaires à Istanbul, est envoyée en pension en Angleterre. Elle y reste jusqu'à la fin du collège puis revient à Istanbul pour le lycée. Une fois diplômée, elle part à Londres poursuivre des études d'histoire, suivie d'un troisième cycle en relations internationales à New York. Elle revient ensuite à Londres, où elle travaille durant un an en tant que consultante pour un institut de recherche international. Fin 2001, cet institut l'envoie en mission à Istanbul pour un an. Elle décide finalement de rester à Istanbul et s'installe dans la maison familiale.

\section{Kaan :}

Kaan est né en 1976 à Izmir. Il est fils unique. Kaan est le seul, parmi les enquêtés, dont les parents sont divorcés. Son père est cadre dirigeant et architecte dans le secteur des bâtiments et travaux publics, et sa mère est professeur de psychologie. Après des études primaires et secondaires à Izmir, il part pour Washington, où il débute des études d'économie avant d'être transféré dans une université mieux cotée de l'Etat de New York. Il travaille ensuite durant quatre ans à New York puis à San Francisco pour une grande multinationale américaine en tant qu'analyste financier. Il rentre en 
Turquie fin 2001, soit environ six mois avant le début de l'enquête. Plutôt que de retourner à Izmir, il décide de s'installer à Istanbul et accepte l'offre d'une entreprise américaine installée en Turquie.

\section{Bulut :}

Bulut est né en 1977 à Paris, mais sa famille est stambouliote. Il a une sœur aînée. Son père est universitaire, mais il a en outre occupé à plusieurs reprises des postes à responsabilité dans la haute fonction publique en Turquie et dans diverses institutions internationales. Bulut vit à Paris avec sa famille jusqu'à l'âge de dix ans, puis à Ankara pour la majeure partie de ses études secondaires, avant de retourner à Paris pour la dernière année de lycée, du fait de l'évolution de la carrière de son père. Il part ensuite suivre des études d'ingénieur à Boston, puis vit à New York durant un an. À son retour en Turquie début 2001, soit un an et demi avant le début de l'enquête, il travaille en tant qu'ingénieur dans un grand groupe turc, puis en tant que chef de projet dans une entreprise de production et d'organisation événementielle. Finalement, passionné de musique électronique, il décide de devenir DJ professionnel et crée son propre label.

\section{Nesli :}

Nesli est née en 1976 à Istanbul. Elle est fille unique. Son père a effectué une carrière dans la haute fonction publique turque et internationale, avant d'ouvrir récemment un cabinet de conseil financier. Nesli a vécu dans le Maryland durant les années d'école primaire, puis à Ankara et à Istanbul durant ses études secondaires. Elle part ensuite poursuivre des études universitaires en Pennsylvanie, à la suite desquelles elle travaille durant cinq années à New York dans la finance, puis dans la production cinématographique. Elle rentre finalement à Istanbul fin 2002, soit environ six mois avant le début de l'enquête. Nesli travaille depuis lors dans le bureau de conseil de son père. Depuis son départ à la retraite, ce dernier a ouvert ce bureau de conseil qui offre ses services aux entreprises étrangères voulant s'implanter en Turquie.

\section{Bilge :}

Bilge est née en 1975 à Bonn, en Allemagne, mais sa famille est stambouliote. Elle est fille unique. Son père étant diplomate, elle a vécu à Paris entre 4 et 8 ans, et à Bruxelles entre 12 et 16 ans. Le reste de son enfance s'est écoulé à Ankara, où les diplomates sont tenus de revenir tous les quatre ans. À la fin du lycée, elle part suivre des études universitaires à Londres, où son père sera d'ailleurs nommé l'année suivante. Bilge reste au total sept années à Londres, où elle travaille également durant deux ans dans le département recherche d'une grande banque. Elle rentre début 2001, soit un peu plus d'un an avant le début de l'enquête. Formée en économie et en informatique, elle décide, au moment de son retour, de devenir photographe.

\section{NOTES}

1. Une étude publiée en 1974 par Erdoğan Soral montre que la majorité des entrepreneurs Turcs de l'époque était d'anciens hauts fonctionnaires ottomans - c'est le cas en particulier de $75 \%$ des 
entrepreneurs qui ont fondé leur entreprise entre 1921 et 1930 - ou d'anciens commerçants et marchands (Soral, 1974). Ces résultats conconcordent avec ceux du travail réalisé par Bates l'année précédente, selon lesquels $77 \%$ des élites économiques de la nouvelle République étaient issues de la fonction publique ou du commerce. Cette dernière étude ajoute en outre que les pères de ces entrepreneurs étaient eux-mêmes fonctionnaires ou marchands en leur temps (Bates, 1973).

2. En se basant sur le Who's Who ? (Kim Kimdir ?) de 1988-89, Ayşe Buğra a montré que 194 des 217 membres d'alors de l'Association des industriels et hommes d'affaires turcs (TÜsİAD) possèdent au moins un diplôme universitaire de second cycle. Elle note en particulier que plus de la moitié des hommes d'affaires nés entre 1910 et 1950 possèdent un diplôme d'ingénieur.

3. Il faut relativiser cette très forte homogénéité d'origine sociale en précisant que les $20 \%$ de liens dont la profession du père est inconnue des enquêtés sont des relations de travail ou des rencontres fortuites, dont on peut supposer que les origines sociales sont plus hétérogènes. Par ailleurs, ces pourcentages ne sont pas également répartis au sein de chaque réseau égocentré. Logiquement, on trouve davantage d'enfants de chefs d'entreprises dans les réseaux de Murat (80 \%) et de Selin ( $85 \%)$, dont les pères sont également chefs d'entreprise. À l'inverse, les réseaux de Bilge et de Nesli, dont les pères ont fait leur carrière dans la haute fonction publique internationale, comportent près de $50 \%$ de liens dont les pères ont suivi des carrières similaires.

4. Il est possible de dégager, à partir de ces 8 réseaux, une "structure de réseau bourgeois ", car l'on y retrouve un certain nombre de caractéristiques communes renvoyant à la socialisation en milieu bourgeois :

- un grand nombre de liens, en particulier de liens anciens: cela renvoie aux compétences propres à la socialisation internationale. Très tôt, on apprend à se constituer un carnet d'adresses international et à conserver un lien sur une longue durée ;

- un taux de densité très élevé et surtout un noyau unique : même les réseaux les plus dissociés possèdent un noyau unique, ce qui est d'autant plus surprenant qu'il s'agit d'individus qui ont vécu dans plusieurs pays différents et ont travaillé dans des secteurs professionnels différents ;

- la famille nucléaire se trouve dans le noyau du réseau: les parents et les frères et sœurs connaissent un grand nombre de liens dans le réseau ( $45 \%$ en moyenne pour les mères, $35 \%$ pour les pères et $40 \%$ pour les frères et soeurs), y compris les amis rencontrés à l'étranger ;

- il n'y a presque pas de points marginaux (contact liés à aucun autre dans le réseau) : cela renvoie à des réseaux distribués, caractéristique propre aux réseaux bourgeois.

5. D'après MEB (2001), Milli Eğitim Sayısal Veriler, Ankara, pp. 266-274, cité par A. Gençler, A. Çolak (2003) op. cit. Les données du Ministère de l'éducation (MEB) et de l'UNESCO diffèrent, et les chiffres avancés sont probablement en dessous de la réalité. L'UNESCO se base sur des données récoltées dans les pays hôtes, si bien que les données dépendent de l'efficacité des systèmes de comptage nationaux. Tandis que pour les États-Unis, par exemple, l'UNESCO peut se référer directement aux statistiques d'organismes spécialisés tels qu'Open Doors, de telles statistiques sur les étudiants étrangers n'existent pas le plus souvent dans des nouvelles républiques telles que l'Azerbaïdjan. Quant aux statistiques du Ministère de l'éducation turc, elles sont obtenues sur place. Ainsi, la fiabilité n'est réelle que pour les garçons, du fait des contrôles douaniers liés aux obligations du service militaire (sur les 22000 étudiants suivant un cursus à l'étranger par leurs propres moyens recensés par le MEB en $2001,90 \%$ sont des hommes).

6. La mode est définie par Blumer comme un phénomène cyclique regroupant l'ensemble des influences et des échanges entre les individus au sein de la société, et participant à la formation et à l'expression des goûts collectifs (Blumer, 1968).

7. Des centaines de "Bureaux de conseil pour l'éducation à l'étranger " ("Yurtdışı eğitim danışmanlığı ») recommandent à leurs clients les universités avec lesquelles ils ont passé un accord leur permettant notamment de faciliter à leurs clients l'obtention du visa de séjour. En outre, les éditions Education Abroad, filiale d'OPTIMA, bureau de conseil spécialisé depuis 1991 
dans l'éducation à l'étranger, publient depuis 2001 un « Guide annuel de l'éducation à l'étranger » et une revue trimestrielle, le « Journal de l'éducation à l'étranger », où les universités étrangères peuvent passer leurs annonces. Hürriyet, l'un des principaux quotidiens nationaux, publie également en supplément un « Guide de l'éducation à l'étranger » depuis 2005. Enfin, l'entreprise A2, filiale d'ASBA, bureau de conseil spécialisé depuis 1996 dans l'éducation à l'étranger, organise depuis 2001 à Istanbul, Izmir et Ankara, une « foire internationale de l'éducation à l'étranger ", à laquelle les universités étrangères viennent participer.

8. Pour les familles de la grande bourgeoisie, l'objectif est d'intégrer les meilleurs lycées privés (kolej) du pays. A Istanbul, le classement n'a guère changé durant les quinze dernières années : le collège américain Robert est suivi du Lycée Allemand et du Lycée Américain d’Üsküdar. Seul un lycée fondé récemment, le Lycée Koç, a réussi à s'immicer parmi l'élite des lycées, en tablant justement sur la préparation à l'international.

9. Originellement «Scholastic Aptitude Test». Aujourd'hui, le sigle a été conservé, mais les initiales n'ont plus cette signification.

10. En particulier les liens avec les conseillers d'orientation américains des meilleurs lycées privés d'Istanbul, tels que le Robert College et le Koç Lisesi, ainsi qu'avec diverses associations telles que le Rotary Club. On retrouve, parmi les élèves du centre, les grands noms de la grande bourgeoisie d'affaires, ainsi que les enfants de la «noblesse d'État».

11. Harvard admet chaque année en moyenne $10 \%$ des candidats au premier cycle (2000 admis pour 20000 applications) et $9 \%$ des étudiants sont étrangers. En comparaison, Brown admet $15 \%$ des candidats et accueille $9 \%$ d'étrangers ; University of Pennsylvania admet $20 \%$ des candidats et accueille $13 \%$ d'étrangers; et University of Virginia (publique) admet $30 \%$ des candidats et accueille $5 \%$ d'étrangers.

12. D'après les statistiques fournies sur les sites de ces universités : www.upenn.edu/about/ facts.php et www.brown.edu/web/facts.shtml

13. Devenue une appellation de prestige se référant aux universités les plus sélectives et les plus anciennes du système universitaire américain, la Ivy League, fondée en 1953, est à l'origine un championnat interuniversitaire sportif (football, baseball, basket-ball, athlétisme, etc.) mis en place par les recteurs de ces universités privées. Il s'agit de Brown, Columbia, Cornell, Dartmouth, Harvard, University of Pennsylvania, Princeton et Yale.

14. 520 / 597 liens sont nés entre 1972 et 1982, et 516/567 (soit $91 \%$ ) si l'on retire les membres de la famille.

15. Cette trajectoire est de loin la plus fréquente parmi les liens présentant cette durée migratoire. Cependant, d'autres « combinaisons » sont bien évidemment possibles. En particulier, certains liens sont partis à l'étranger pour suivre un mastère puis sont restés travailler une ou plusieurs années avant de rentrer en Turquie. Il faut également prendre en considération le fait que plus d'un tiers des 597 liens de nationalité turque ayant vécu à l'étranger se trouvaient toujours à l'étranger au moment de l'enquête.

16. Le nombre de visas de travail temporaires H1-B est limité depuis l'Immigration Act de 1990. Alors fixée à 65.000 visas par an, la limite a été portée à 115.000 visas par an en 1998 .

17. Entretien réalisé le 22 juin 2004 dans les locaux de TIPPS.

18. En revanche, Nermin Abadan-Unat, qui fut la première à décrire des formes de retour volontaires et planifiées en Turquie, a rompu avec le regard exclusivement pessimiste porté par l'approche structurelle, dont elle s'était pourtant inspirée. Abadan-Unat considère que les discriminations dont les travailleurs rentrés d'Allemagne entre 1973 et 1985 ont été victimes durant leur migration - un tiers d'entre eux disent que c'est la principale raison qui les a motivé à rentrer - ont fait d'eux des citoyens plus exigeants. Pour elle, ces Almancı de la première et de la seconde génération (migrants et enfants de migrants) représentent une "nouvelle synthèse ", et qu'ils exercent un impact significatif sur la vie économique et politique turque (Abadan-Unat, 1996). 
19. Cette définition renvoie à la définition scientifique de la résonance: «Lorsque la vibration excitatrice périodique imprimée à un système physique est égale à la fréquence naturelle de ce système, l'amplitude de vibration de ce système augmente sensiblement : le système est dit alors en résonance ». McGraw-Hill Encyclopedia of Science \& Technology (2002), McGraw-Hill, 9 éd., « resonance », p. 455.

\section{RÉSUMÉS}

Cet article est issu d'une recherche doctorale portant, entre autres aspects, sur les mobilités des enfants de la grande bourgeoisie turque vers l'étranger. La mobilité, qui peut parfois prendre l'allure d'une migration d'assez longue durée, a pour objectif la formation à l'étranger, particulièrement aux États-Unis et dans quelques pays européens, des enfants de la bourgeoisie d'affaires et de la bureaucratie, civile et militaire, qui formeront la future élite de la Turquie moderne. Cette pratique, déjà ancienne en Turquie, a pris ces dernières années une tournure nouvelle et plus générale ; elle montre comment la mobilité ou la migration pour études permet aux futurs cadres et élites de se former à l'étranger dans l'optique d'un retour socialement et économiquement valorisé, loin de la définition habituelle de l'exode des cerveaux. L'article s'appuie sur un travail de représentation par graphes des réseaux construits entre la Turquie et le reste du monde, souvent centré sur les États-Unis.

International means of social reproduction. Migratory paths for Turkish upper class members. The present paper originates in a doctoral piece of research which analyzes, among other points, the mobility of young people belonging to the upper class to foreign countries. Such mobility may, in some cases, be considered as a migration process over a rather long period of time. It is primarily meant to complete the education and training abroad, especially in the United States and some European countries, of children of the business bourgeoisie and the civilian and military bureaucracy, who are the future elite of modern Turkey. That practice has existed for a long time in Turkey, but it took, in recent years, a new and more generalized turn. It highlights the way in which mobility, or migration for studies, allows the future higher executives and elite to be educated abroad, with the prospect of a socially and economically enhanced status, and it has nothing to do with the usual definition of brain drain. The paper is based upon a representation in graphs of the networks built between Turkey and the rest of the world, and frequently focused upon the United States.

Las vías internacionales de la reproducción social. Trayectorias migratorias de la gran burguesía turca. Este artículo deriva de una investigación doctoral que se centra, entre otros aspectos, en la movilidad hacia el extranjero de los niños de la gran burguesía turca. El objetivo de dicha movilidad, que puede en ocasiones convertirse en una emigración de gran duración, es de formar en el extranjero (particularmente en Estados Unidos y en varios países europeos) a los niños de la burguesía de negocios y de la burocracia civil y militar; niños que formarán parte de la futura élite de la Turquía moderna. Esta práctica, ya anciana en Turquía, ha tomado estos últimos años una nueva orientación: la movilidad o la emigración por estudios permite a los futuros altos cargos y elites formarse en el extranjero bajo la óptica de un retorno social y económicamente revalorizado y muy diferente de la definición habitual de la fuga de cerebros. El 
artículo se apoya sobre un trabajo de representación grafica de redes que ligan Turquía al resto del mundo redes que se centran a menudo en Estados Unidos.

INDEX

Index géographique : Turquie

Mots-clés : élite, étudiants, formation, mobilité géographique, réseaux sociaux, réseaux transnationaux, Turcs

\section{AUTEUR}

\section{DAVID BEHAR}

Docteur en Sociologie, École des Hautes Études en Sciences Sociales, Paris, david.behar@ehess.fr 